\title{
Crossover from contact propagation to chemical propagation in first-passage percolation
}

\author{
Alan R. Kerstein and Boyd F. Edwards \\ Sandia National Laboratories, Livermore, California 94550
}

(Received 9 September 1985)

\begin{abstract}
On lattices whose bonds are assigned time delays from a bimodal distribution with modes at $b$ and $a \gg b$ whose relative weights are $p$ and $1-p$, the dependence of the first-passage velocity $v$ on $p$ is investigated by means of scaling arguments and computations. As $p$ increases, $v$ exhibits a sharp rise near the percolation threshold due to a crossover from the contact-propagation regime, in which slow-bond crossings are rate limiting, to the chemical-propagation regime, in which the tortuosity of the shortest path through the fast-bond network is rate limiting. Previous analyses of criticality in the limit $a / b \rightarrow \infty$ are extended by obtaining corrections to scaling for finite $a / b$. The qualitative picture is confirmed by small-cell real-space renormalization-group (RSRG) computations, and proposed scaling laws for critical exponents are tested by means of large-cell Monte Carlo RSRG computations and by a computational method analogous to the transfer matrix for conductivity. The development is analogous to the well-known theory of the conductivity of a disordered medium, and in fact corresponds to a particular limit of the nonlinear conductivity problem. This correspondence, in conjunction with the proposed scaling laws and a duality argument, is exploited in order to evaluate critical exponents governing two-dimensional superfluid flow. Scaling arguments developed here are shown to be applicable also to stirred percolation, leading to a new scaling law for the conductivity of stirred-percolation systems.
\end{abstract}

\section{INTRODUCTION}

The "chemical distance" between two points or regions $A$ and $B$, defined as the length of the shortest connecting path entirely contained within the allowed region of a disordered medium, has recently been recognized as an important descriptor of the physical as well as the geometrical properties of the medium. ${ }^{2,3}$ In particular, a scaling law relating the fractal dimension of the shortest path across a percolation cluster to the critical behavior of the first-passage velocity $v$ through a percolating network has been derived. ${ }^{2,4}$ The vanishing of $v$ at the percolation threshold $p_{c}$ of the medium is analogous to the vanishing of the conductivity, ${ }^{5}$ if we take the allowed region to be the conducting region.

We have noted ${ }^{6}$ that this analogy can be extended by considering the first-passage analogue of the random superconducting network. ${ }^{5,7}$ In the domain $p<p_{c}$ of the conductivity problem, the superconducting region consists of isolated clusters, with the overall conductivity governed by the time for charge carriers to cross short normalconducting paths spanning the gaps between large superconducting clusters. In the first-passage analogue, formulated on a square bond lattice for concreteness, bonds have zero time delay with probability $p$ and finite time delay otherwise, representing a regime in which the crossing of "slow" bonds spanning the gaps between "fast" clusters is rate limiting with respect to the first-passage velocity. We call this the contact-propagation ${ }^{6,8,9}$ regime, to distinguish it from the "chemical-propagation" regime above $p_{c}$. In the contact-propagation regime, there is no simple relationship between chemical distance and first-passage velocity. Nevertheless, a scaling law relating the critical behavior of the first-passage velocity to a geometrical ex- ponent has been proposed. ${ }^{6}$ This and the scaling law for the chemical-propagation regime provide a framework for predicting and interpreting the critical exponents on either side of $p=p_{c}$, although unsolved problems remain, as we shall indicate.

Here, we combine and extend previous results concerning the criticality of $v$ above and below $p_{c}$ in order to obtain a unified picture of the $p$ dependence of $v$. After reviewing and extending previous results concerning criticality on either side of $p_{c}$, we examine the crossover from contact propagation to chemical propagation under the assumption that the slow and fast bonds are assigned finite time delays $a$ and $b \ll<$, respectively. Chemical propagation corresponds to the limit $a \rightarrow \infty$ with $b$ fixed. As noted previously, ${ }^{6}$ the contact-propagation regime is associated with a shift to the slow time scale, i.e., the limit $b \rightarrow 0$ with $a$ fixed. The crossover between regimes is obtained by taking $a / b$ to be large but finite so that, as $p_{c}$ is approached from either side, a transition regime is encountered in which $v$ depends on the ratio $a / b$. Scaling predictions for the transition regime are obtained by arguments analogous to those for the conductivity problem. ${ }^{5}$ We next consider the distribution dependence of the critical exponent for chemical propagation, applying a heuristic variational method ${ }^{10}$ to a particular class of distributions governing the fast-bond time delays. We conclude the scaling analysis by exploiting the equivalence of firstpassage percolation to a nonlinear conductivity problem, in conjunction with the proposed scaling laws and a duality argument, in order to evaluate critical exponents governing two-dimensional superfluid flow.

To confirm the qualitative picture, $v(p)$ is computed for all $0 \leq p \leq 1$ by means of the real-space renormalization-group (RSRG), using a small-cell 
method. Away from $p_{c}$, we compute $v(p)$ from the small-cell recursion relations using a renormalizationgroup method. ${ }^{11,12}$ Below $p_{c}, v(p)$ is computed for finite $a / b$ as well as for $a / b=\infty$.

Details of the large-cell Monte Carlo RSRG method which we previously used ${ }^{6}$ to compute the critical exponent for the contact-propagation regime are provided, and new results for the chemical-propagation regime are presented. A computational method analogous to the transfer matrix ${ }^{13}$ for conductivity is used to verify the predicted finite- $(a / b)$ scaling in the transition regime. Our previously reported estimates ${ }^{14}$ of the chemicaldistance exponent obtained from the latter computation are discussed. Finally, the relationship of the firstpassage problem analyzed here to the scaling regimes of stirred percolation ${ }^{8,9,15}$ is exploited to obtain scaling laws governing propagation and transport in stirred-percolation systems.

\section{SCALING PREDICTIONS FOR THE FIRST-PASSAGE VELOCITY}

\section{A. Propagation exponents}

The first-passage velocity $v$ is defined as the large- $R$ limit of the ratio $R / \tau_{A B}$, where $\tau_{A B}$ is the first-passage time from $A$ to $B$, and $R$ is the Euclidean distance from $A$ to $B \cdot{ }^{2,6,16} v$ as thus defined is directly applicable to the modeling of signal transmission processes in biological and other applications in which the first arrival per se is the feature of interest. ${ }^{2}$ As implied by the terminology chemical distance and chemical propagation, $v$ has also been interpreted as a measure of the rate of chemical conversion associated with the propagation of a chemical reaction front through a disordered medium.

In the chemical-propagation regime $p>p_{c}$, with fast bonds assigned time-delay unity and slow bonds assigned infinite time delay, the first-passage velocity vanishes near $p_{c}$ as $v \sim\left(p-p_{c}\right)^{\theta}$, where $\theta$ obeys the scaling law ${ }^{2,4}$

$$
\theta=v(\phi-1) \text {, }
$$

in which $v$ is the correlation-length exponent and the chemical-distance exponent $\phi$ (in the notation of Ref. 6) governs the $R$ dependence of the first-passage time, i.e., $\tau_{A B} \sim R^{\phi}$ for $R$ less than the correlation length $\xi$. This result was derived by assuming that $\tau_{A B}$ is independent of $p-p_{c}$ for $R \ll \xi$ and that $\tau_{A B}$ is proportional to $R$ for $R \gg \xi$. The derivation is thus analogous to the derivation of the scaling law for diffusion on percolation clusters, ${ }^{17}$ except that for the diffusion problem, $\tau_{A B}$ is proportional to $R^{2}$ for large $R$, the constant of proportionality being the inverse of the diffusion coefficient. As in the diffusion problem (or in the conductivity problem, which is equivalent based on the Einstein relation ${ }^{18}$ ), the scaling law relates a fractal index governing geometrical fine structure to an exponent governing a large-scale process, in this case propagation. In the chemical-propagation regime, the propagation exponent $\theta$ is the analogue of the conductivity exponent ${ }^{5} t$.

We have noted ${ }^{6}$ that the analogy may be extended to the contact-propagation regime in which bonds have zero time delay with probability $p<p_{c}$ or finite time delay otherwise. Analogous to the divergence of the conductivity of the random superconducting network with critical exponent $s$, we obtained the scaling form $v \sim\left(p_{c}-p\right)^{-\psi}$ for contact propagation, where the propagation exponent $\psi$ obeys the scaling law

$$
\psi=v(\tilde{\phi} / k+1) .
$$

$\widetilde{\phi}$ is the fractal dimension of finite-delay (slow) bonds common to the perimeters of two large clusters of zerodelay (fast) bonds, and the cumulative distribution $F(x)$ governing the finite time delays is assumed to obey $F(x) \sim x^{k}$ for small $x$. We further proposed that $\tilde{\phi}$ is related to the fractal dimension $d_{u}$ of the "unscreened" bonds ${ }^{7}$ on a percolation cluster according to

$$
\widetilde{\phi}=d_{u}+d_{h}-d \text {, }
$$

where $d_{h}$ is the fractal dimension of the exterior perimeter ("hull") of a percolation cluster. ${ }^{19}$ The quantity $d_{h}-d$ manifests the distinction between the propagation process, to which cluster crossings within an internal hole of a fast cluster do not contribute, and the diffusion process, to which all cluster crossings contribute.

Equation (2.2) was derived by analogy to the derivation $^{7,18}$ of the diffusivity $D$ of the "de Gennes termite," the charge carrier on the random superconducting network. Namely, we started from

$$
v \sim \xi / \tau_{A B},
$$

where $\tau_{A B}$ is the mean time for first passage from the fast cluster containing point $A$, to the fast cluster containing a point $B$ a Euclidean distance $\xi \sim\left(p_{c}-p\right)^{-v}$ from $A$. We then employed a result from the sampling theory of order statistics ${ }^{20}$ to obtain

$$
1 / n=F\left(\tau_{A B}\right)
$$

where $n \gg 1$ is the number of slow bonds common to the perimeters of the fast clusters $A$ and $B$. Under the assumption that $F(x) \sim x^{k}$ for small $x$, this gave $\tau_{A B} \sim n^{-1 / k}$. Finally, the scaling assumption $n \sim \xi^{\tilde{\phi}}$ gave Eq. (2.2), with the proposed scaling, Eq. (2.3), for $\widetilde{\phi}$.

An alternative to Eq. (2.3) has been proposed by $Y$. Kantor $^{21}$ based on an extension of Coniglio's ${ }^{22}$ theorem for the number density of cutting bonds. We briefly outline Kantor's derivation. Coniglio considered the scaling of the average number $L$ of cutting bonds between two points $i$ and $j$ separated by a distance $\xi$ on an incipient infinite cluster (IIC). (A cutting bond is a bond whose removal breaks the connection between $i$ and $j$.) For $\epsilon=p_{c}-p \ll 1$, Coniglio showed that $L=(p / S) d S / d p$, where the mean cluster size $S$ is of order $\epsilon^{-\gamma}$, and thus $L \sim \epsilon^{-1}$. He obtained this by determining the reduction of the probability $p_{i j}$ that $i$ and $j$ are connected due to a reduction of $p$ by $d p$, attributing the reduction of $p_{i j}$ to the removal of cutting bonds. Now we consider points $i$ and $j$, separated by a distance $\xi$, on distinct IIC's. For such points, the increase of $p_{i j}$ due to an increase of $p$ by $d p$ is attributable to the addition of crossing bonds, i.e., bonds common to the perimeters of the two IIC's. The derivation of the scaling of the number $n$ of such bonds 
parallels Coniglio's derivation of the scaling of $L$, giving $n \sim \epsilon^{-1} \sim \xi^{1 / \nu}$. Since $\widetilde{\phi}$ is defined in the derivation of Eq. (2.2) by the relation $n \sim \xi^{\tilde{\phi}}$, this gives

$$
\widetilde{\phi}=1 / v \text {, }
$$

so that Eq. (2.2) becomes

$$
\psi=v+1 / k \text {. }
$$

For $d=2$, Eq. (2.6) gives $\widetilde{\phi}=0.75$, in comparison to the value $0.73 \pm 0.01$ given by $\mathrm{Eq}$. (2.3). [The uncertainty of the latter value is due to the uncertainty of quantities appearing in Eq. (2.3).] Both results are consistent with Monte Carlo RSRG computational estimates discussed in Sec. IV B. These results indicate that the quantity $d_{u}$, which obeys the scaling law ${ }^{7} d_{u}=d-2+s / v$, corresponds to a set of bonds which is larger than the set of crossing bonds. In fact, it is unclear whether this exponent can be associated with an identifiable set of bonds governing the scaling regime of the random superconducting network. ${ }^{7}$ For the propagation problem, the relevant bonds are the crossing bonds, governed by Eq. (2.6). Furthermore, the agreement of Eq. (2.6) with the computational estimates indicates that the distinction between fast-cluster crossings at internal holes and at the external perimeter is irrelevant to lowest order in the scaling regime of the propagation problem. Were this not the case, a term such as $d_{h}-d$ would be needed in Eq. (2.6) to achieve agreement with the computational estimates.

Equations (2.6) and (2.7) are remarkable in that the contact-propagation exponent is expressed in terms of the correlation-length exponent $v$ and a parameter governing the time-delay distribution. Thus, no new exponents need be introduced in order to characterize this scaling regime. In contrast, no comparable expression for the chemicaldistance exponent $\phi$ appearing in the scaling law for $\theta$ has yet been established, as discussed in Sec. V C. Likewise, comparable expressions for the conductivity exponents $s$ and $t$ have not yet been established, although proposals have been offered. ${ }^{7}$

We now consider the scaling of $v$ near $p_{c}$ for distributions $F(x)$ that do not vanish as a power of $x$ for small $x$. In this case, the inversion of Eq. (2.5) to obtain the large- $n$ behavior of $\tau_{A B}$ does not yield a power-law dependence. Therefore, $v$ is no longer a power of $\xi$. We now have

$$
v \sim \xi / F^{-1}\left(\xi^{-\tilde{\phi}}\right)
$$

where $F^{-1}$ denotes a functional inverse of the cumulative distribution. For instance, if $F(x) \sim(-x / \ln x)^{k}$ for small $x$, then in the large- $n$ limit, Eq. (2.5) can be inverted to obtain $\tau_{A B} \sim n^{-1 / k} \ln n$, which gives $v \sim \xi^{\widetilde{\phi} / k+1} / \ln \xi$. Thus, a logarithmic factor in the denominator of $F(x)$ introduces a logarithmic factor in the denominator of the scaling law for $v$. If $F(x) \sim \exp (-1 / x)$, which vanishes faster than any power of $x$ for small $x$, then we obtain $v \sim \xi \ln \xi$, which diverges more slowly than any finite- $k$ case governed by Eq. (2.7), but not as slowly as the case $k \rightarrow \infty$, which corresponds to $F(x)$ vanishing in some neighborhood of $x=0$. (In the latter limit, we obtain $\psi=v$. This result applies, for instance, to the case in which all slow-bond time delays are identical.) Thus, various scaling forms for $v$ may be obtained in the contact-propagation regime, determined from the small- $x$ behavior of $F(x)$ by implementing the functional inversion in Eq. (2.8).

\section{B. Crossover and corrections to scaling}

We consider the implications of these results with regard to the dependence of $v$ on $p$ if we assume that the mean delay for slow bonds is equal to $a$, while the fastbond time delays all have a small finite value $b \ll a$. (The case $b=0$ was considered in Sec. II A.) This formulation is analogous to the generalization of the conductivity problem in which a large but finite value is assigned to the ratio of the mean conductivities of "metallic" and "dielectric" bonds. 5,23

Specializing to a square lattice with lattice spacing unity, and assuming that bonds are independently assigned to be fast or slow with probabilities $p$ and $1-p$ respectively, $v$ is necessarily an increasing function of $p$, with $v(1)=1 / b$. As $p$ approaches $p_{c}$ from below, we anticipate a sharp rise in $v$ near $p_{c}$, corresponding to the divergence associated with the contact-propagation regime. Above $p_{c}$, we expect the sharp rise to continue for a short $p$ interval, corresponding to the criticality associated with the chemical-propagation regime. The degree to which these features are evident for a given finite value of $a / b$ will now be addressed. The analysis is restricted to slowbond time-delay distributions $F(x)$ which vanish as a power of $x$ for small $x$, so that the contact-propagation scaling law, Eq. (2.2), is applicable. The method of analysis is likewise applicable to the more general situation in which Eq. (2.8) is employed to obtain the scaling of $v$ in the contact-propagation regime, but the analysis must then be implemented on a case-by-case basis.

Three features associated with crossover are analyzed. First, we estimate the values $p_{-}<p_{c}$ and $p_{+}>p_{c}$ for crossover to the transition regime from the contactpropagation and chemical-propagation regimes, respectively. Second, we estimate the dependence of $v\left(p_{c}\right)$ on the ratio $a / b$. Finally, we derive corrections to scaling associated with crossover to the transition regime.

Previously, ${ }^{14}$ we analyzed the transition regime by analogy to Straley's ${ }^{24}$ homogeneous-function representation of the conductivity problem. Alternatively, Straley's finitesize scaling analysis ${ }^{5}$ of the conductivity crossover can be carried over to the propagation problem. We proceed here in a somewhat different fashion in order to indicate the variety of different approaches by which the transition regime can be analyzed.

For finite $a / b$, the first-passage time $\tau_{A B}$ from point $A$ to point $B$ may be expressed as the sum $\tau_{A B}=\tau_{f}+\tau_{s}$ of contributions due to the crossing of fast and slow bonds, respectively. In the contact-propagation regime, the $\mathrm{Eu}$ clidean distance $\xi$ is typically traversed by crossing the order- $\xi^{\phi}$ fast bonds comprising the shortest path across a fast cluster, and then crossing one slow bond, so that $\tau_{f} \sim b \xi^{\phi}$ and $\tau_{s} \sim a \xi^{-\tilde{\phi} / k}$. (The latter result follows from the scaling arguments of Sec. II A.) Crossover occurs when $\xi$ is large enough so that slow-bond crossings are no longer rate limiting, i.e., $\tau_{f} \sim \tau_{s} . p_{-}$is determined from 
the value of $\xi \sim\left(p_{c}-p_{-}\right)^{-v}$ for which the latter condition is satisfied. Using Eqs. (2.1) and (2.2) to express the result in terms of the propagation exponents, we obtain

$$
p_{c}-p_{-} \sim(b / a)^{1 /(\theta+\psi)} .
$$

In the chemical-propagation regime, crossover occurs when $\xi$ is large enough so that it is "occasionally" advantageous to bypass a tortuous path through the percolating network of fast bonds by crossing a slow bond. If there are $j$ slow-bond crossings per traversal of a Euclidean distance $\xi$ between points $A$ and $B$, then the Euclidean distance between successive slow-bond crossings is of order $\xi / j$, assuming that the minimum-time path is homogeneous (i.e., nonfractal) over distances greater than $\xi / j$. Taking $\xi / j$ to be the effective correlation length, the time to traverse the fast-bond network between successive slowbond crossings is $\tau_{f}(j) \sim b(\xi / j)^{\phi}$ and the time to traverse each slow bond is $\tau_{s}(j) \sim a(\xi / j)^{-\tilde{\phi} / k}$. (These expressions are obtained by replacing $\xi$ with $\xi / j$ in the previous scalings for $\tau_{f}$ and $\tau_{s}$.) The Euclidean distance $\xi$ between points $A$ and $B$ is therefore traversed in a total time

$\tau_{A B} \sim j\left[\tau_{f}(j)+\tau_{s}(j)\right] \sim j b(\xi / j)^{\phi}+j a(\xi / j)^{-\tilde{\phi} / k}$.

$j$ is as yet undetermined. Since we are seeking the minimum-time path, we minimize this expression for $\tau_{A B}$ with respect to $j$. Again using Eqs. (2.1) and (2.2), we obtain

$$
j \sim \xi(b / a)^{v /(\theta+\psi)},
$$

which gives

$$
\tau_{A B} \sim a \xi(b / a)^{\psi /(\theta+\psi)} .
$$

Crossover occurs when $\xi$ is large enough so that $j$ is of order unity. Setting $j=1$ in Eq. (2.11) and proceeding as before, we obtain

$$
p_{+}-p_{c} \sim(b / a)^{1 /(\theta+\psi)} .
$$

Equations (2.9) and (2.13) indicate that $\left|p-p_{c}\right|$ $<(b / a)^{1 /(\theta+\psi)}$ is the transition regime.

Equation (2.12) is valid wherever $j \geq 1$, i.e., in the transition regime. Therefore, in the transition regime, and in particular at $p_{c}$, we obtain

$$
v\left(p_{c}\right) \sim \xi / \tau_{A B} \sim(1 / b)(b / a)^{\theta /(\theta+\psi)} .
$$

Finally, we consider corrections to scaling as the transition regime is approached. In the contact-propagation regime, we derive the lowest-order correction with respect to the small quantity $\tau_{f} / \tau_{s}$. Using our expressions for these quantities and expressing the final result in terms of the propagation exponents, we obtain

$$
\begin{aligned}
v & \sim\left(\xi / \tau_{s}\right)\left(1-\tau_{f} / \tau_{s}\right) \\
& \sim(1 / a)\left(p_{c}-p\right)^{-\psi}\left[1-(b / a)\left(p_{c}-p\right)^{-\theta-\psi}\right] .
\end{aligned}
$$

In the chemical-propagation regime, no analogous correction is obtainable in the framework of scaling theory, because slow bonds are not crossed except in localities where the tortuosity of the percolating fast-bond network is greater than the scaling value.

The results for crossover and corrections to scaling,
Eqs. (2.9), (2.13), (2.14), and (2.15), have been expressed in terms of the propagation exponents in order to highlight the correspondence between these results and finite- $(a / b)$ effects in the conductivity problem. Substituting the conductivity exponents $s$ and $t$ for $\psi$ and $\theta$, respectively, and substituting $1 / a$ and $1 / b$ for $a$ and $b$, respectively, (since $a$ and $b$ represent bond conductances rather than bond resistances in the conductivity literature), we find that Eqs. (2.9) and (2.13) are equivalent to Straley's ${ }^{5}$ Eq. (3.4), Eq. (2.14) is equivalent to his Eq. (3.5), and Eq. (2.15) is equivalent to the second line of his ${ }^{25} \mathrm{Eq}$. (6) (except for a sign error in the latter). However, the absence of a chemical-propagation correction to scaling analogous to the conductivity result [the first line of Straley's ${ }^{25}$ Eq. (6)] indicates that the propagation and conductivity problems are formally equivalent in some but not all respects. The relationship between the two problems is discussed further in Sec. II D.

\section{Distribution dependence of the chemical-propagation exponent}

Although we have characterized the scaling of $v$ in the contact-propagation regime for an arbitrary distribution $F(x)$ of slow-bond time delays, scaling in the chemicalpropagation regime $(a=\infty)$ is as yet characterized only in the case that all fast-bond time delays have the same value, here denoted as $b$. In the chemical-propagation regime, $v$ for given $p$ is either unchanged or reduced if the fast-bond delays are independently distributed (with mean value $b$ ) rather than identically equal to $b$. To show this, we consider the (not necessarily unique) minimum-time path from $A$ to $B$ for a given realization of the case of fast-bond delays all equal. In this case, $\tau_{A B}=b l$, where $l$ is the number of bonds in the path. If the fast-bond delays for this realization are instead independently distributed (with mean value $b$ ), then in the large- $l$ limit, the crossing time along that same path is again $b l$. However, there now exists the possibility that some other path of length $l^{\prime}>l$ has a mean fast-bond delay less than $b l / l^{\prime}$, so that $\tau_{A B}<b l$ along the path $l^{\prime}$. For any distribution of fast-bond delays, this will occur for some realizations. Whether this will result in a decrease in the ensembleaveraged first-passage velocity $v$ depends on the frequency and the magnitude of the reductions in $\tau_{A B}$.

Lacking an exact method to address this problem, we adopt a heuristic approach based on a variational method previously applied ${ }^{10}$ to the conductivity problem. Specifically, we assume that the fast-bond time-delay distribution is of the form

$$
h(x)=\left\{\begin{array}{l}
0, \quad x<1 \\
(\beta-1) x^{-\beta}, \quad x \geq 1,
\end{array}\right.
$$

where $1<\beta<2$. (The analysis which follows is valid only for $\beta$ in this range.) $v$ is approximated by first separating the fast bonds into two groups: fast bonds for which $x \geq x_{0}$, and those for which $x<x_{0}$, where the value $x_{0}$ is determined shortly by a variational procedure. For the first group, the time delays are set equal to infinity, i.e., they are redesignated as slow bonds. For the second 
group, the time delays are all set equal to their mean value conditioned on $x<x_{0}$, i.e., the time delay

$$
\tau_{0}=\frac{\int_{0}^{x_{0}} x h(x) d x}{\int_{0}^{x_{0}} h(x) d x}=\frac{\beta-1}{2-\beta} \frac{x_{0}^{2-\beta}-1}{1-x_{0}^{1-\beta}},
$$

is assigned to each bond in this group. The fraction of bonds designated as fast is now

$$
p_{0}=p \int_{0}^{x_{0}} h(x) d x=p\left(1-x_{0}^{1-\beta}\right) \text {. }
$$

The effect of these modifications is to increase the first-passage time $\tau_{A B}$, since the average fast-bond delay along the true minimum-time path will be less than the conditionally averaged time delay, Eq. (2.17). For the modified time-delay distribution, Eq. (2.1) is applicable, so the modified propagation velocity $v_{0}$ obeys the scaling

$$
v_{0} \sim \tau_{0}^{-1}\left(p_{0}-p_{c}\right)^{\theta}
$$

for $p_{0} \gtrsim p_{c}$, where $v_{0}$ is smaller than the true propagation velocity $v$. To minimize the error introduced by the modifications, we choose the value of $x_{0}$ which maximizes $v_{0}$. Substituting Eqs. (2.17) and (2.18) into Eq. (2.19), we solve $d v_{0} / d x_{0}=0$ for $x_{0}$, which depends on $p$, $\theta$, and $\beta$. To lowest order in $\left(p-p_{c}\right)$, Eqs. (2.17) and (2.19) then give

$$
v_{0} \sim\left(p-p_{c}\right)^{\theta+(2-\beta) /(\beta-1)} .
$$

To compare this result to the variational estimate of the distribution-dependent conductivity exponent, the appropriate changes of variables are $\theta \rightarrow t$ and $\beta \rightarrow 2-\alpha$, which yields Eq. (4.5) of Kogut and Straley. ${ }^{10}$ For $\beta$ within its allowed range $1<\beta<2$, Eq. (2.20) indicates that the effect of the assumed distribution, Eq. (2.16), is to increase the value of the chemical-propagation exponent.

Alternative methods ${ }^{26,27}$ for estimating the $\beta$ dependence of the conductivity exponent $t$ for the assumed distribution, Eq. (2.16), each give different results, indicating the likelihood that none of the methods is exact. Therefore, the variational estimate derived here serves primarily to demonstrate that the chemical-propagation exponent may be distribution dependent. This leaves open the broader question of the precise scaling form of $v$ in the chemical-propagation regime for an arbitrary distribution of fast-bond time delays. In this regard, the characterization of the chemical-propagation regime is less complete at present than the characterization of the contactpropagation regime, discussed in Sec. II B.

\section{Equivalence to a conductivity problem}

If the time delays in the first-passage problem are reinterpreted as bond resistances in an electrical network, then the minimum-time path, whose traversal time is the firstpassage time $\tau_{A B}$, is also the lowest-resistance path from $A$ to $B$. It has been noted ${ }^{28}$ that this path determines the onset voltage for current flow in the small- $\alpha$ limit of the nonlinear resistor network in which the current-voltage $(I-V)$ characteristic for a bond with finite resistance $r$ is

$$
V=r I^{\alpha},
$$

where we take $I$ to be positive. Based on this observation, we derive relationships between the propagation exponents $\theta$ and $\psi$ and the small- $\alpha$ values of the $\alpha$-dependent nonlinear conductivity exponents $t(\alpha)$ and $s(\alpha)$, respectively, for which scaling laws have been obtained. ${ }^{28}$

We first consider the chemical-propagation regime, seeking a relationship between $\theta$ and $t(\alpha)$. We assign resistance $r=1$ to a fraction $p \gtrsim p_{c}$ of bonds on a $d$ dimensional network (chosen to be hypercubic for concreteness) of edge $L$, with the remaining bonds assigned infinite resistance. In the small- $\alpha$ limit, the $I-V$ characteristic of each bond is that of a bipolar Zener diode, which allows current to flow only above a threshold voltage $V_{0}=r=1$. A voltage $U$ is applied across two opposite faces $A$ and $B$ of the hypercube, and we seek to determine the threshold value $U_{0}$ for current flow. Current will flow only if every bond along some path from $A$ to $B$ has a voltage drop of at least unity. The smallest value of $U$ for which this condition is satisfied corresponds to a voltage drop of unity across every bond along the (not necessarily unique) shortest path from $A$ to $B$. Given our interpretation of time delays as bond resistances, the threshold value $U_{0}$ of the total voltage drop is therefore equal to the first-passage time $\tau_{A B}$.

We now exploit the feature of the $I-V$ characteristic, Eq. (2.21), which originally motivated the analysis of this functional form. Namely, any network whose elements have this $I-V$ characteristic has the same $I-V$ characteristic, with $r$ replaced by an effective resistance $r_{\text {eff }}$ which depends on the resistances of the individual elements and the network geometry. In particular, a network of bipolar Zener diodes is again a bipolar Zener diode, where the threshold voltage is given by $U_{0}=r_{\text {eff }}$. For the hypercubic network, we therefore have

$$
r_{\text {eff }}=\tau_{A B} \text {. }
$$

For given $\alpha$, the application of Eq. (2.21) to our network gives $r_{\text {eff }}=U / I^{\alpha}$. Substituting this into the constitutive relation,

$$
\rho_{\mathrm{eff}} \sim(U / L)\left(I / L^{d-1}\right)^{-\alpha},
$$

for the effective resistivity $\rho_{\text {eff }}$ of the hypercubic network of nonlinear conductors, ${ }^{28}$ we obtain

$$
\rho_{\text {eff }} \sim r_{\text {eff }} L^{(d-1) \alpha-1} .
$$

For length scales $L$ equal to or greater than the correlation length $\xi$, the network is homogeneous, so the firstpassage time is given by $\tau_{A B}=L / v$, where $v \sim\left(p-p_{c}\right)^{\theta}$ is the first-passage velocity near criticality in the chemicalpropagation regime. In conjunction with Eqs. (2.22) and (2.24), we obtain

$$
\rho_{\text {eff }} \sim\left(p-p_{c}\right)^{-\theta} L^{(d-1) \alpha},
$$

which is valid only in the small- $\alpha$ limit which was assumed in the derivation of Eq. (2.22). We compare this to the previously derived ${ }^{28}$ result for the effective resistivity on length scales up to $\xi$,

$$
\rho_{\mathrm{eff}} \sim\left(p-p_{c}\right)^{-\alpha t(\alpha)},
$$

where $t(\alpha)$ governs the divergence of the effective con- 
ductivity $\quad \sigma_{\text {eff }} \sim\left(p-p_{c}\right)^{t(\alpha)}$ for $\quad p \gtrsim p_{c}$. Taking $L=\xi \sim\left(p-p_{c}\right)^{-v}$ in Eq. (2.25) and comparing Eq. (2.26), we obtain

$$
\alpha t(\alpha)=\theta+(d-1) \alpha v .
$$

Equation (2.27) has been derived only for the limit $\alpha \rightarrow 0$, for which we obtain $\alpha t(\alpha) \rightarrow \theta$. However, the $O(\alpha)$ term has been retained on the right-hand side based on the expectation that the analysis is valid to this order for small but finite $\alpha$.

To justify this expectation, we compare Eq. (2.27) to a proposed scaling law for $t(\alpha)$ which is motivated by the Skal-Shklovskii-de Gennes (SSDG) model ${ }^{5,28-30}$ for the conducting network just above the percolation threshold. In the SSDG model, the network is represented near criticality as a collection of effectively one-dimensional resistor chains, connected at nodes with average separation $\xi$. Assuming that the effective resistance of a typical resistor chain diverges as $r_{\mathrm{eff}} \sim\left(p-p_{c}\right)^{-\zeta}$, where $\zeta$ depends on $d$ and $\alpha$, the scaling law

$$
\alpha t(\alpha)=\zeta-v+(d-1) \alpha v
$$

has been derived. ${ }^{28}$

In the small- $\alpha$ limit, the bonds of a typical resistor chain are bipolar Zener diodes so, as before, the effective resistance $r_{\text {eff }}$ is determined by the shortest conducting path between chain ends $A$ and $B$. Therefore, $r_{\text {eff }}=\tau_{A B} \sim \xi^{\phi} \sim\left(p-p_{c}\right)^{-\nu \phi}$, where we have invoked the SSDG assumption that $A$ and $B$ are separated by a Euclidean distance $\xi$. This gives $r_{\text {eff }} \sim\left(p-p_{c}\right)^{-v \phi}$ for the typical resistor chain. Thus, we obtain the anticipated correspondence $\xi_{0}=v \phi$, where the subscript indicates that $\zeta$ is evaluated at $\alpha=0$. In other words, (2.28) reduces to Eq. (2.27) in the limit $\alpha \rightarrow 0$, lending support to the validity of Eq. (2.27) for small but finite $\alpha$. Our derivation shows that the small- $\alpha$ result can be obtained without invoking the geometrical assumptions of the SSDG model.

The derivation of the analogue of Eq. (2.27) for the contact-propagation regime with $r=1$ for all finiteresistance bonds is essentially the same, except that the scaling forms are now $\xi \sim\left(p_{c}-p\right)^{-v}, v \sim\left(p_{c}-p\right)^{-\psi}$, and $\rho_{\text {eff }} \sim\left(p_{c}-p\right)^{\alpha s(\alpha)}$. Therefore, the scaling law for $s(\alpha)$ is obtained by making the substitutions $\theta \rightarrow-\psi$ and $t \rightarrow-s$ in Eq. (2.27), giving

$$
\alpha s(\alpha)=\psi-(d-1) \alpha v \text {. }
$$

Again, this has been derived only for the limit $\alpha \rightarrow 0$, but the $O(\alpha)$ term has been retained to suggest a possible finite- $\alpha$ generalization.

No such generalization has been proposed, but a relationship between $s(\alpha)$ and $t(\alpha)$ has been obtained for $d=2$ by a duality argument. The duality relationship ${ }^{28}$

$$
\alpha s(\alpha)=t(1 / \alpha),
$$

in conjunction with Eq. (2.28), gives

$$
\alpha s(\alpha)=v+\alpha[\zeta(1 / \alpha)-v]
$$

for $d=2$, where the $\alpha$ dependence of $\zeta$ is now indicated explicitly.

Comparison of Eqs. (2.29) and (2.31) in the limit $\alpha \rightarrow 0$ gives $\psi=v$, a result which was shown at the end of Sec. II $A$ to be valid for arbitrary $d$ for slow-bond delays all identical. If we assume Eqs. (2.28) and (2.30) to be correct to $O(\alpha)$, then we can equate the $O(\alpha)$ terms for $d=2$. We obtain $\zeta(\infty)=0$, implying that the resistance of a typical resistor chain is independent of $p$ near criticality for $\alpha=\infty$. This result is correct because $\alpha=\infty$ corresponds to a network of saturating resistors ${ }^{28}$ in which current flows without dissipation (i.e., no voltage drop) up to a critical current at which the flow is choked. (This model also represents superfluid flow in a random network with a limiting flow velocity. ${ }^{31}$ ) In such a network, the resistance of a typical resistor chain is determined by the largest bond resistance in the chain, which is independent of chain length and therefore independent of $p$ in this instance because we have assumed that all finite-resistance bonds have the same resistance. Thus, Eq. (2.29) as well as Eq. (2.27) is found to be consistent to order $\alpha$ with results previously derived using the SSDG model.

We consider the implications of these results for criticality in superfluid flow. $t(\infty)$ is the exponent governing the vanishing of the saturation current, above which the maximum allowed velocity for superfluid flow is exceeded and therefore the flow is choked. ${ }^{28}$ From Eqs. (2.29) and (2.30) we obtain

$$
t(1 / \alpha)=\psi-(d-1) \alpha v
$$

for $\alpha \rightarrow 0$. In particular, $t(\infty)=\psi$. Based on our earlier result $\psi=v$, we have thus evaluated the exponent governing the critical vanishing of superfluid flow in twodimensional random networks, for which only a rough estimate was previously available. ${ }^{31}$

Thus far we have considered only the case in which the finite-resistance bonds all have identical resistance. However, the derivation of Eq. (2.29) is valid not only in the aforementioned special case (for which $\psi=v$ ), but generally for finite-resistance distributions for which the scaling law for $\psi$, Eq. (2.7), is applicable, namely distributions which obey $F(x) \sim x^{k}$ for small $x$. The duality principle, according to which $t$ is obtained from $s$ by taking $\alpha \rightarrow 1 / \alpha$ and reinterpreting the resistance distribution as a conductance distribution, ${ }^{28}$ similarly generalizes. Therefore, we obtain

$$
t(\infty)=v+1 / k
$$

a result which may be of practical consequence with regard to criticality of superfluid flow, since the exponent depends not only on the toplogy of the flow network, as previously recognized, ${ }^{31}$ but also on the distribution of flow resistances of individual elements in the network.

Alternatively, one may consider a two-dimensional flow network in which a fraction $p<p_{c}$ of flow channels is wide enough to accommodate currents up to the saturation value with negligible flow velocity. The remaining fraction $1-p$ of the flow channels provides the flow resistance. Arguments analogous to the derivation of Eq. (2.33) then lead to the identification of $\theta$ as the critical exponent governing the divergence of the saturation current as $p$ approaches $p_{c}$. The crossover analysis of Sec. II B is likewise applicable. 


\section{E. Finite-size scaling}

We now consider the $p$ dependence of the first-passage velocity $v$ from edge $A$ to the opposite edge $B$ of a lattice in the limit $R \rightarrow \infty$, as before, but now the lattice is assumed to have finite $\operatorname{span} L \gg 1$ in all transverse directions. On such a lattice, the fast bonds do not percolate from $A$ to $B$ for $p<1$. Slow bonds must therefore be crossed in order to traverse the lattice, so in the limit $a / b \rightarrow \infty$, slow-bond crossings are rate limiting for all $p<1$. [Finite- $(a / b)$ effects are discussed at the end of this section.] We therefore analyze the propagation problem for finite $L$ by taking $b=0$ and assuming, as in Sec. II $\mathrm{A}$, that the slow-bond time delays are governed by a cumulative distribution $F(x)$ with the parameter $k$ defined as before. We specialize to a two-dimensional strip of width $L$, since the analysis is readily generalized to higher dimensions.

The analysis closely parallels the finite-size scaling analysis for stirred percolation. ${ }^{9}$ In that analysis, the lattice was viewed as a row of $L \times L$ boxes. The key step was the estimation of the number $h$ of adjacent boxes typically spanned by a fast-bond cluster, since this determines the forward advance of the propagation front per cluster contact event. [Here, $L$ corresponds to $b$, and $h$ to $1 /\left(1-p_{\text {conn }}\right)$, of the previous analysis.] Only the discrete-time process was considered (the stirredpercolation analogue of the limit $k \rightarrow \infty$; see Sec. VI A), so $v$ was proportional to $h L$. The analysis of the firstpassage percolation problem for $k \rightarrow \infty$ (corresponding, for instance, to the case in which all slow-bond time delays are identical) is formally identical to the previous analysis, so the results carry over directly. To generalize to finite $k$, we need to estimate the time $\tau$ between contact events in order to estimate $v \sim h L / \tau$. (For $k \rightarrow \infty, \tau$ is of order unity for all $L$ and $p$.)

As shown in Sec. II A, $\tau \sim n^{-1 / k}$, where $n$ is now the number of slow bonds common to the perimeters of fast clusters $A$ and $B$ on the two-dimensional strip. We noted earlier that, on a lattice which is unbounded in all directions, $n \sim \xi^{\widetilde{\phi}}$, where $\widetilde{\phi}$ is given by Eq. (2.6). Here we adopt the scaling hypothesis, validated for other fractal indices governing percolation clusters, ${ }^{32,33}$ that the quantity $n$ is fractal (i.e., self-similar) on length scales less than $\xi$, and homogeneous (i.e., it scales with Euclidean dimension) on length scales greater than $\xi$. In particular, since $\xi$ diverges at $p_{c}, L<\xi$ in some neighborhood of $p_{c}$. Therefore, the scaling hypothesis implies $n \sim L^{\widetilde{\phi}}$ in this neighborhood, so we obtain $\tau \sim L^{-\tilde{\phi} / k}$. On the other hand, for $p-p_{c}$ positive and of order unity, $\xi$ is of order unity, ${ }^{32}$ so $L>\xi$. Therefore, the scaling hypothesis implies $n \sim L$ in this domain, giving $\tau \sim L^{-1 / k}$.

Combining these results and the previous results ${ }^{9}$ for the dependence of $h$ on $L$ and $p$, we obtain the following three finite-size scaling regimes for $v$. At $p_{c}$, the $L$ dependence of $v$ is given by

$$
v \sim L^{1+\tilde{\phi} / k},
$$

since $h$ is of order unity at $p_{c}$. For $p-p_{c}$ and $1-p$ both positive and of order unity,

$$
\begin{aligned}
v \sim L^{1+1 / v+1 / k} \exp [ & \pi L^{2 / v}\left(p-p_{c}\right)^{2} \\
& \left.-2 \pi a L^{1 / v}\left(p-p_{c}\right)\right],
\end{aligned}
$$

where $a$ is a lattice-dependent quantity defined in the stirred-percolation analysis. Finally, in some neighborhood of $p=1$,

$$
v \sim L^{1+1 / k}(1-p)^{-L},
$$

which is valid not only for large $L$, but for $L$ of order unity as well.

If we now take $a / b$ to be finite, crossover from contact propagation to chemical propagation must occur for some $p<1$ because, in the absence of finite- $(a / b)$ effects, $v$ diverges near $p=1$, according to Eq. (2.36). In fact, the crossover can occur at any $p_{c} \leq p<1$, depending on the values of the governing parameters. We derive the criterion for crossover at $p_{c}$ and we briefly indicate how the criteria for crossover at larger $p$ can be obtained.

Crossover occurs at $p_{c}$ if slow-bond crossings are no longer rate limiting, i.e., $\tau_{f} \geq \tau_{s}$ in the notation of Sec. II B. At $p_{c}$, it follows from the scaling hypothesis that $\tau_{f} \sim b L^{\phi}$ and $\tau_{s} \sim a L^{-\tilde{\phi} / k}$. Using Eqs. (2.1) and (2.2), we find that the criterion for crossover at $p_{c}$ is $a / b \leq L^{(\theta+\psi) / v}$.

For $p$ far enough above $p_{c}$ so that $\xi$ is of order unity, fast-bond clusters are homogeneous so $\tau_{f} \sim b h L$. Likewise, $\tau_{s} \sim a L^{-1 / k}$. The crossover criterion is therefore $a / b \leq h L^{1+1 / k}$. Substitution of the functional forms governing the dependence of $h$ on $L$ and $p$ in the respective scaling regimes above $p_{c}$ gives the explicit forms of the crossover criteria for these regimes. Thus, depending on the magnitudes of the parameters $a / b$ and $L$, as many as five scaling regimes may be obtained: contact propagation below $p_{c}$, three finite-size regimes at and above $p_{c}$, and finally chemical propagation.

\section{SMALL-CELL RSRG COMPUTATIONS}

\section{A. Scaling near the percolation threshold}

The real-space renormalization group has proven useful for computing geometrical and transport properties of disordered networks. ${ }^{34}$ Here we use the RSRG to compute $v(p)$ and to demonstrate various qualitative and quantitative aspects of the predicted scalings.

For small-cell RSRG computations, we adopt the usu$\mathrm{al}^{34-36}$ eight-bond Kadanoff cell for bond percolation on a square lattice with length rescaling parameter $L=2$. For purposes of determining the first-passage time from one edge of the cell to the opposite edge, this cell is equivalent to a five-bond Wheatstone bridge (Fig. 1 inset). Each bond is assigned either a time delay $b$ with probability $p$ or a time delay $a \gg b$ with probability $1-p$. By determining the first-passage time $\tau$ for each of the $2^{5}$ configurations of time-delay assignments on the Wheatstone bridge, we obtain the probability distribution given in Table I.

By analogy to the small-cell RSRG for the conductivity problem, ${ }^{37}$ we might proceed by assigning time delays drawn from this distribution to bonds of the renormalized lattice and then constructing a new probability distribu- 


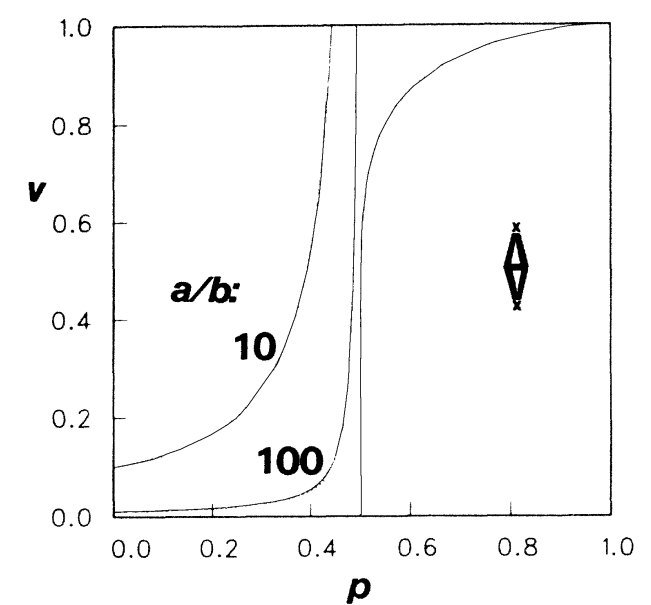

FIG. 1. First-passage velocity $v$ versus fraction $p$ of fast bonds (time delay $b=1$ ) on the square lattice, computed from recursion relations for an eight-bond Kadanoff cell, equivalent to a five-bond Wheatstone bridge (inset). $v(p)$ is shown for slow-bond time delays $a=10$ and $a=100$, respectively, with finite- $(a / b)$ effects omitted (solid curves) and included (dashed curves).

tion, analogous to Table $I$, for the first-passage time on a five-bond Wheatstone bridge of renormalized bonds. We could then deduce the critical exponents from the shifts of the peaks of this (bimodal) distribution upon successive iterations. However, for $a \gg b$ little error is introduced by approximating the renormalized time-delay distribution given in Table $I$ by two $\delta$ functions located at $b^{\prime}=\langle\tau \mid \tau=O(b)\rangle / L$ and $a^{\prime}=\langle\tau \mid \tau=O(a)\rangle / L$, where the rescaling of the bond length by the factor $L=2$ has been included. (With this length rescaling, the renormalized propagation velocity $v^{\prime}$ is simply the inverse of the renormalized first-passage time $\tau^{\prime}$.) The relative weights of the renormalized time delays $b^{\prime}$ and $a^{\prime}$ are $p^{\prime}$ and $1-p^{\prime}$, respectively, where $p^{\prime}(p)$ is the usual renormalized-bond probability, given by the recursion relation

$$
p^{\prime}=2 p^{5}-5 p^{4}+2 p^{3}+2 p^{2}
$$

for the five-bond Wheatstone bridge. ${ }^{34-36}$ Now, the cell renormalization is implemented by updating the parameter set $\{a, b, p\}$ to the renormalized set $\left\{a^{\prime}, b^{\prime}, p^{\prime}\right\}$. Using Table I to evaluate $a^{\prime}$ and $b^{\prime}$, we obtain the additional recursion relations

$$
a^{\prime}=\left[(a+b) p(1-p)^{2}\left(2-p^{2}\right)+a(1-p)^{4}\right] /\left(1-p^{\prime}\right),
$$

TABLE I. Probability distribution of first-passage time for a five-bond Wheatstone bridge. Bond time delay is $b$ with probability $p$ or $a$ with probability $1-p$.

\begin{tabular}{ll}
\hline \hline First-passage time & \multicolumn{1}{c}{ Probability } \\
\hline $2 b$ & $p^{2}\left(2-p^{2}\right)$ \\
$3 b$ & $2 p^{3}(1-p)^{2}$ \\
$a+b$ & $2 p(1-p)^{2}\left(2-p^{2}\right)$ \\
$2 a$ & $(1-p)^{4}$ \\
\hline \hline
\end{tabular}

$$
b^{\prime}=b\left[1+p^{3}(1-p)^{2} / p^{\prime}\right]
$$

Equation (3.1b) was derived previously ${ }^{9}$ for the special case $b=0$. Equation (3.1c) has also been obtained by Ohtsuki and Keyes, ${ }^{38}$ who investigate the chemicalpropagation regime using an approach similar to ours.

For $p$ below the unstable fixed point $p^{*}=\frac{1}{2}$ of Eq. (3.1a), successive iterations converge to the stable fixed point $p=0$, indicating that the weight of the $b$-bond contribution to the bond distribution vanishes. This result is expected since this domain corresponds to the contactpropagation regime $p<p_{c}\left(=\frac{1}{2}\right.$ for the square lattice) in which crossings of the slow (type- $a$ ) bonds determine scaling of the propagation velocity. However, the recursion relation for $a$ is coupled to the recursion relation for $b$, reflecting the fact that $b$ bonds as well as $a$ bonds are crossed in the contact-propagation regime. This coupling will enable us to compute finite- $(a / b)$ effects.

Similarly, we find that the weight of the $a$-bond contribution to the bond distribution vanishes upon successive iterations for $p>\frac{1}{2}$, the domain of attraction of the fixed point $p=1$. Since the recursion relation for $b$ depends only on $b$ and $p$, the recursion relation for $a$ is irrelevant in this domain. This is reasonable for $a=\infty$ because slow bonds are not crossed in the chemical-propagation regime. The absence of $a$ dependence for finite $a$ indicates that finite- $(a / b)$ effects in the chemical-propagation regime are omitted, a consequence of the approximation of the distribution given in Table $I$ by two $\delta$ functions. [This approximation subsumes finite- $(a / b)$ effects, which stem from the $(a+b)$ term in the distribution, into the recursion relation for $a$.] This is another manifestation of the observation in Sec. II B that corrections to scaling for chemical propagation are not obtained in the framework of scaling theory.

In the limit $a / b \rightarrow \infty$, we can set $b=0$ in the recursion relation for $a$, so that the right-hand side of Eq. (3.1b) is proportional to $a$. Thus, the $a$ and $b$ recursion relations are decoupled from each other in this limit. We use these recursion relations to estimate the propagation exponents $\psi$ and $\theta$, respectively. To do this, we use the result of Hong and Stanley ${ }^{36}$ that the critical exponent $y$ for any percolation quantity $Q(p)$ which scales as $Q(p) \sim\left|p-p_{c}\right|^{y}$ is given by

$$
y=-\ln \lambda_{Q} / \ln \lambda_{p},
$$

where $\lambda_{Q}=\partial Q^{\prime} /\left.\partial Q\right|_{p=p^{*}}$ and $\lambda_{p}=\partial p^{\prime} /\left.\partial p\right|_{p=p^{*}}$ are derivatives of the recursion relations $Q^{\prime}(Q, p)$ and $p^{\prime}(p)$, evaluated at $p^{*}$. Taking $Q$ to be $a$ in the contactpropagation and $b$ in the chemical-propagation regime, Eq. (3.2) governs the criticality of the first-passage time in the respective regimes. Since the length-rescaled firstpassage time is the inverse of $v, y=\psi$ in the contactpropagation regime and $y=-\theta$ in the chemicalpropagation regime. Therefore,

$$
\psi=-\left.\frac{\ln \left(\partial a^{\prime} / \partial a\right)}{\ln \left(\partial p^{\prime} / \partial p\right)}\right|_{p=p^{*}}
$$




$$
\theta=\left.\frac{\ln \left(\partial b^{\prime} / \partial b\right)}{\ln \left(\partial p^{\prime} / \partial p\right)}\right|_{p=p^{*}},
$$

where $a^{\prime}(a)$ and $b^{\prime}(b)$ are given by the first-passage-time recursion relations, Eqs. (3.1b) and (3.1c), with $b=0$ in (3.1b).

Evaluating these expressions, we obtain the estimates $\psi=\ln \left(\frac{16}{9}\right) / \ln \left(\frac{13}{8}\right)=1.19$ and $\theta=\ln \left(\frac{17}{16}\right) / \ln \left(\frac{13}{8}\right)=0.12$ for $d=2$ based on the eight-bond Kadanoff cell. For $d=3$, a similar analysis performed for a simple-cubic lattice using a 24-bond Kadanoff cell with $L=2$ gives $\psi=0.76$ and $\theta=0.18$. Given the approximations inherent in the small-cell RSRG method, ${ }^{34}$ these results are in rather good agreement with the scaling prediction $\psi=v\left(=\frac{4}{3}\right.$ for $d=2$ and 0.88 for $d=3$ ) (Ref. 39) and with the computed results $^{2} \theta=0.13$ for $d=2$ and 0.31 for $d=3$. (In Sec. VC, however, we obtain $\theta$ values which differ from these.)

\section{B. Propagation velocity away from criticality}

The recursion relations (3.1a)-(3.1c) serve not only to estimate the propagation exponents, but also to estimate the dependence of $v$ on $p$ for all $0<p<1$. The method, applicable in principle to any dynamical quantity which scales near $p_{c}$, was demonstrated in recent analyses of geometrical $^{11}$ and transport ${ }^{11,12}$ properties of a random network. The method consists of numerical iteration of the coupled recursion relations for $p$ and for the dynamical quantity until $p$ is close enough to a stable fixed point so that further iterations give negligible increments. The iterations begin at the unrenormalized values of $p$ and of the dynamical quantity. The method is based on the premise that the small-cell RSRG embodies roughly the correct dependence of the correlation length on $p$ (as evidenced by the fairly accurate estimates of the correlationlength exponent $v$ obtained using small cells ${ }^{35}$ ). Successive iterations renormalize the dynamical quantity until the correlation length is reduced to the lattice spacing. At this stage, the system is homogeneous, so further iterations in conjunction with length rescaling [as incorporated into the recursion relations (3.1a)-(3.1c)] have no effect. The final value of the dynamical quantity is therefore interpreted as its effective macroscopic value corresponding to the original value of $p$.

This method has been employed to compute $v(p)$ for $d=2$ in the limit $a / b \rightarrow \infty$ for the contact-propagation regime, using (3.1a) and (3.1b) with $b=0$, and for the chemical-propagation regime, using (3.1a) and (3.1c). The results are the solid curves of Fig. 1. (The curve to the right of $p=\frac{1}{2}$ is the same as that obtained by Ohtsuki and Keyes. ${ }^{38}$ ) Since the propagation velocity is of order $1 / a$ in the contact-propagation and of order $1 / b$ in the chemical-propagation regime, finite values must be arbitrarily assigned to $a$ and $b$ in order to plot the two regimes on the same axes. In Fig. 1, we take $b=1$, which fixes the scale for the chemical-propagation regime. In the contact-propagation regime, two curves differing only by a scale factor are shown, corresponding to $a=10$ and $a=100$, respectively. The results of analogous computations for $d=3$, based on recursion relations for the 24 -

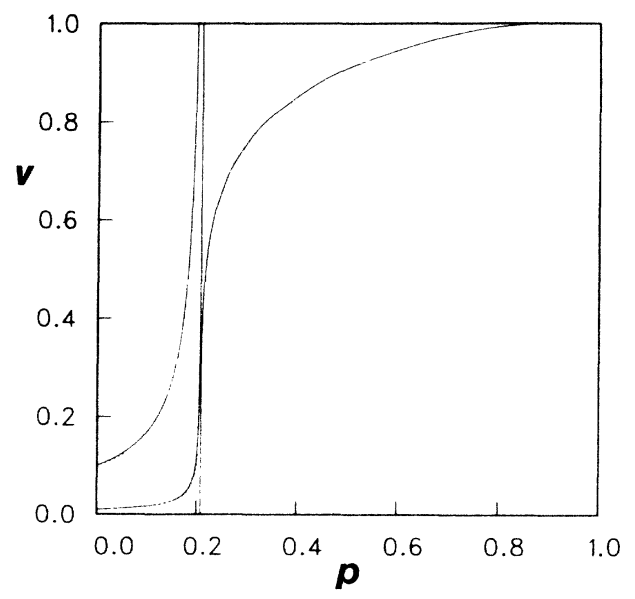

FIG. 2. First-passage velocity $v$ versus fraction $p$ of fast bonds (time delay $b=1$ ) on the simple-cubic lattice, computed from recursion relations for a 24-bond Kadanoff cell. $v(p)$ is shown for slow-bond time delays $a=10$ and $a=100$, respectively, with finite- $(a / b)$ effects omitted (solid curves) and included (dashed curves).

bond Kadanoff cell of the simple-cubic lattice, are shown in Fig. 2. For this cell, we obtain the unstable fixed point $p^{*}=0.21$, which in this instance differs from the bond percolation threshold $p_{c}=0.2492$ for the simple-cubic lattice. $^{40}$

The results are qualitatively similar to Fig. 1 of Efros and Shklovskii, ${ }^{23}$ which shows the dependence of the effective conductivity of a random network on the relative proportions of metallic and dielectric bonds. The similarity reflects the close correspondence of the propagation and conductivity problems, discussed in Sec. II.

The solid curves in Figs. 1 and 2 demonstrate the divergence (vanishing) of $v$ in the contact (chemical) propagation regime. Near $p_{c}, v(p)$ exhibits the scalings analyzed in Sec. III A, with critical exponents whose values are equal to the estimates of that section, because the computational method is mathematically equivalent near $p_{c}$ to the analysis which gave Eqs. (3.3) and (3.4). The apparent nonmonotonicity of $v(p)$ is a consequence of the fact that corrections to scaling for finite $a / b$ were not included in the recursion relations used to compute these curves. As indicated by the analysis of Sec. II B, the uncorrected curves for the contact and chemical-propagation regimes are upper and lower bounds, respectively, on the finite$(a / b)$ curve. Thus, $v(p)$ for finite $a / b$ is a monotonic function constrained to lie between the solid curves. Even without a corrections-to-scaling analysis, Figs. 1 and 2 indicate that this constraint assures a sigmoidal (" $S$ shaped") dependence of $v$ on $p$. In Sec. III C, we estimate the dependence of the $v(p)$ curve on the numerical value of the ratio $a / b$.

At $p=1$, the $p$ derivative of $v$ vanishes, while this derivative is nonzero at $p=0$. This is due to the vanishing of the $p$ derivative of the $b$ recursion relation, Eq. (3.1c), at $p=1$, in contrast to the nonzero value of the $p$ derivative of the $a$ recursion relation, Eq. (3.1b), at $p=0$. $v$ is insensitive to $p$ near $p=1$ because the few slow bonds 
which are present have essentially no impact on the tortuosity of the minimum-time path through the fast-bond network. Near $p=0$, the few fast bonds which are present determine the preferential paths and therefore influence $v$.

\section{Sensitivity to the time-delay ratio}

The method employed in Sec. III B to compute $v(p)$ for $a / b=\infty$ can also be used for finite values of the timedelay ratio $a / b$. The recursion relations are iterated as before, but instead of taking $b=0$ in Eq. (3.1b), the recursion relations are initialized by taking $b=1$ [consistent with the velocity normalization $v(1)=1$ ] and taking $a$ to be the value of the time-delay ratio. As before, the recursion relation (3.1b) determines $v$ in the contactpropagation regime, while (3.1c) determines $v$ in the chemical-propagation regime. However, (3.1b) and (3.1c) are now coupled through the dependence of the $a$ recursion relation on $b$, so all three recursion relations must be iterated simultaneously in the contact-propagation regime. The $b$ recursion relation, though, does not depend on $a$, so the finite value of $a / b$ does not enter into the chemicalpropagation computation, as noted in Sec. II A.

Focusing therefore on the contact-propagation regime, we caution that Eqs. (3.1a)-(3.1c) were derived under the assumption that the renormalized time-delay distribution consists of two widely separated peaks which can be approximated by $\delta$ functions at $a^{\prime}$ and $b^{\prime}$. Since the effect of renormalization is to shift the peaks closer, ${ }^{37}$ the ratio $a^{\prime} / b^{\prime}$ is reduced with each iteration, so it is necessary to check whether the final (fixed-point) value of this ratio is large enough to assure that the use of Eqs. (3.1a)-(3.1c) is valid. We adopt the criterion, that after each iteration, the ordering of the magnitudes of the first-passage times in the probability distribution of Table I (or the corresponding distribution for $d=3$ ) must be preserved. For the five-bond Wheatstone bridge, violation of this ordering occurs for $3 b^{\prime} \geq a^{\prime}+b^{\prime}$, so we require $a^{\prime} / b^{\prime}>2$.

The results of computations for $a / b=10$ and $a / b=100$ are indicated by the dashed curves in Figs. 1 and 2. Each curve spans the range of $p$ for which the validity criterion is obeyed. These curves can be extrapolated visually until they meet the chemical-propagation curve, providing an indication of the shape of the $v(p)$ curve. As we would expect from the scaling analysis of crossover, in particular Eq. (2.9), the $p$ interval over which the dashed curves differ significantly from the corresponding solid curves [which omit finite- $(a / b)$ effects] becomes narrow as $a / b$ increases.

\section{LARGE-CELL MONTE CARLO RSRG COMPUTATIONS}

\section{A. Computational method}

The small-cell RSRG computations of Sec. III provide a qualitative picture of the dependence of $v$ upon $p$. To obtain estimates of the propagation exponents with controlled precision, we employ the large-cell Monte Carlo RSRG method. Since the rationale of the method is discussed in detail elsewhere, ${ }^{34,41}$ we outline the method briefly in the context of the present application.

The recursion relations $(3.1 \mathrm{a})-(3.1 \mathrm{c})$ for the square lattice were derived based on rescaling of a cell of edge $L=2$ lattice spacings to a unit cell. This rescaling neglects relevant couplings because the shortest path connecting opposite edges of such a cell embedded in the square lattice may not be entirely contained within the cell. Furthermore, spurious couplings are introduced because the time spent traversing cell edges is neglected, causing the passage time along some paths to be underestimated. As in the case of ordinary percolation, we anticipate that both of these problems are mitigated by choosing a $\mathrm{Ka}$ danoff cell with $L \gg 1$. Now, the available paths within the cell constitute a more representative sample of the totality of available paths, the more so because the minimum-time path is likely to be less tortuous than a "typical" path.

As in the small-cell computations, each bond is assigned either a time delay $b$ with probability $p$ or a time delay $a \gg b$ with probability $1-p$. Again the renormalized time-delay distribution is approximated by two $\delta$ functions located at $b^{\prime}=\langle\tau \mid \tau=O(b)\rangle / L$ and $a^{\prime}=\langle\tau \mid \tau=O(a)\rangle / L$, where the indicated averages are taken over configurations of the $L \times L$ cell. The weights of the renormalized time delays $b^{\prime}$ and $a^{\prime}$ are $p^{\prime}$ and $1-p^{\prime}$, respectively, where $p^{\prime}(p)$ is the recursion relation for ordinary percolation on the $L \times L$ cell. $\left[p^{\prime}(p)\right.$ has been computed for site percolation on the square ${ }^{41}$ and triangular ${ }^{42}$ lattices for a range of $L$ values.]

Provided that these recursion relations can be evaluated, the derivation of Eqs. (3.3) and (3.4) is again applicable, thus providing estimates of the propagation exponents for given $L$. However, evaluation of $\tau$ for all possible configurations is infeasible for large $L$ because the number of configurations is of order $2^{2 L^{2}}$. To overcome this difficulty, a Monte Carlo method is employed in which $\tau$ is evaluated for a randomly sampled subset of the allowed configurations.

A computational realization of the $L \times L$ box is generated by randomly assigning bonds a time delay $b$ with probability $p^{*}$ or $a$ with probability $1-p^{*}$. As before, the recursion relations $a^{\prime}(a)$ and $b^{\prime}(b)$ are proportional to $a$ and $b$, respectively, in the limit $a / b \rightarrow \infty$, since $a$ and $b$ fix the time scales for the contact-propagation and chemical-propagation regimes, respectively. Therefore, the quantity $\left.\left(\partial a^{\prime} / \partial a\right)\right|_{p=p^{*}}$ in Eq. (3.3) is estimated by $\langle\tau \mid \tau=O(a)\rangle / L$, where the expectation is taken over those realizations for which at least one slow bond must be crossed in order to traverse the cell horizontally. Similarly, the quantity $\left.\left(\partial b^{\prime} / \partial b\right)\right|_{p=p^{*}}$ in Eq. (3.4) is estimated by $\langle\tau \mid \tau=O(b)\rangle / L$, where the expectation is taken over those realizations for which there is a connected path of $b$ bonds spanning the cell horizontally. (The horizontalspanning criterion corresponds to connectivity rule $R_{1}$ of Ref. 41.)

Since $\partial a^{\prime} / \partial a$ and $\partial b^{\prime} / \partial b$ in Eqs. (3.3) and (3.4), respectively, need be evaluated only at $p=p^{*}$, the simulations need involve only realizations at this value of $p$. Generally, the fixed point $p^{*}$ of the recursion relation $p^{\prime}(p)$ for an $L \times L$ cell is $L$ dependent and must be evaluated for each $L$ by means of Monte Carlo estimates of $p^{\prime}(p)$ over a 
range of $p$ values. However, the latter computation is not needed in this case because $p^{*}$ is exactly $\frac{1}{2}$ for bond percolation on an $L \times L$ cell of the square lattice. ${ }^{43,44}$ For given $L$, the evaluation of $\left.\left(\partial p^{\prime} / \partial p\right)\right|_{p}$ in the denominators of Eqs. (3.3) and (3.4) also requires computations over a range of $p$ values, but here we need only the latticeindependent large- $L$ asymptotic $\left.\operatorname{result}^{41}\left(\partial p^{\prime} / \partial p\right)\right|_{p^{*}}$ $=L^{1 / v}$.

Substituting these results into Eqs. (3.3) and (3.4), we obtain

$$
\psi=-v[\ln \langle\tau \mid \tau=O(a)\rangle / \ln L-1]
$$

and

$$
\theta=v[\ln \langle\tau \mid \tau=O(b)\rangle / \ln L-1] .
$$

The conditioned means are evaluated for given $L$ by evaluating $\tau$ for a set of realizations of an $L \times L$ cell of the square lattice on which $a$ and $b$ bonds are equally probable, where $a=1$ and $b=0$ for contact propagation [Eq. (4.1)], and $a=\infty$ and $b=1$ for chemical propagation [Eq. (4.2)]. The numerical evaluation of $\tau$ for a given realization is efficiently implemented by the method of "burning,"45 adapted to the present configuration.

Using this method, the propagation exponents can be estimated for $L$ values of order $10^{2}$ or greater, depending on the precision desired and the available computational resources. Though the errors introduced by the finite-cell biases described earlier decrease with increasing $L$, they generally exceed the achievable statistical precision even at $L$ values of this magnitude. These biases can be mitigated, however, by extrapolating the results obtained for various finite $L$ values to $L=\infty$. Based on a simple model of the $L$ dependence of the bias effects, it was originally proposed ${ }^{41}$ that the estimated critical exponent depends linearly on $1 / \ln L$ for large $L$, permitting a straightforward extrapolation to $L=\infty$ on a plot of, say, $\psi$ or $\theta$ versus $1 / \ln L$. It has since been shown that the dependence may be quadratic, ${ }^{42}$ or may have a more complex form. ${ }^{46}$ Details of the extrapolation procedure employed here are presented in Sec. IV B.

The extrapolation procedure is predicated on the assumption that finite-cell biases vanish in the large- $L$ limit, so that the propagation exponents could in principle be determined to arbitrary accuracy, limited only by statistical error, from Eqs. (4.1) and (4.2) in this limit. This implies that the right-hand side of each of these equations should converge to a constant value in the large- $L$ limit, thus providing unbiased estimates of the propagation exponents.

Finally, we note that the first-passage velocity for the present configuration, in which the first passage from edge $A$ to opposite edge $B$ of a square box is sought, is not $a$ priori equivalent to the first-passage velocity for the configuration usually considered, ${ }^{2}$ in which $A$ and $B$ are points. However, the equivalence of the former, "line-toline" process to the latter, "point-to-point" process with respect to first-passage velocity has recently been rigorously established. ${ }^{47}$ In fact, the box need not be square, provided that its aspect ratio is finite in the large$L$ limit. The first-passage velocity is thus sensitive to the distribution of time delays but not to the boundary configuration, facilitating the application of scaling analysis to the problem.

\section{B. Application to contact propagation}

The large-cell Monte Carlo RSRG estimates, Eqs. (4.1) and (4.2), for the propagation exponents were derived by analogy to the small-cell analysis of Sec. III A. The small-cell analysis was limited to networks for which bond time delays take one of two values, $a$ or $b$. This limitation was motivated first, by the simple, explicit form of the first-passage-time distribution (Table I) which was thus obtained, and second, by the validity of repeatedly iterating the recursion relations $(3.1 \mathrm{a})-(3.1 \mathrm{c})$ in this case. (Repeated iteration was employed in Sec. III B to compute the propagation velocity away from criticality.)

The large-cell Monte Carlo RSRG method for estimating the propagation exponents does not require an explicit first-passage-time distribution since the random sampling procedure ensures the correct distributional weighting. Furthermore, the method does not involve iteration of the recursion relations since the expressions for the critical exponents require only that the recursion relations be evaluated at $p^{*}=\frac{1}{2}$. Therefore, the method is no less applicable to more general time-delay distributions. In particular, in the contact-propagation regime we again assign a value $b=0$ to the fast bonds, but the slow bonds may be assigned time delays drawn from any cumulative distribution $F(x)$ with mean value $a$ of order unity. Provided that the slow-bond time delays are sampled from this distribution, Eq. (4.1) provides an estimate of the value of $\psi$ corresponding to this distribution.

For general $\boldsymbol{F}(\boldsymbol{x})$, comparison of the scaling law for $\psi$, Eq. (2.2), and the large-cell RSRG estimate, Eq. (4.1), indicates the physical interpretation of the large- $L$ convergence condition stated at the end of Sec. IV A, namely,

$$
\widetilde{\phi} / k=-\ln \langle\tau \mid \tau=O(a)\rangle / \ln L
$$

in the large- $L$ limit. This is consistent with the result

$$
\tau_{A B} \sim \xi^{-\tilde{\phi} / k}
$$

stated earlier, where $\tau_{A B}$ is the first-passage time between points $A$ and $B$ on distinct fast clusters [so that $\tau_{A B}=O(a)$ ], and the correlation length $\xi$ has the value $L$ at $p=p^{*}$, according to finite-size scaling. ${ }^{41}$ Thus, Eq. (4.3) is simply the finite-size formulation of Eq. (4.4), so the convergence condition will be satisfied provided that the scaling arguments used to derive Eq. (2.2) are valid.

Computed estimates of $\widetilde{\phi}$ for $d=2$ using Eq. (4.3) were presented previously ${ }^{6}$ for the cases of all slow-bond time delays equal, corresponding to $k=\infty$, and three alternative slow-bond time-delay distributions, with $k$ values ranging from 0.2 to 2 . The data for the finite- $k$ distributions are shown in Fig. 3. $(-\widetilde{\phi}$ is plotted for consistency with the format of Fig. 2 of Ref. 6.) The numerical parameters of the exponential distribution $F(x)=1$ $-\exp (-x / 0.95)$, for which $k=1$, and the distribution

$$
F(x)=(2 / \pi) \arctan (x / 0.81)^{k},
$$

with $k$ chosen to be 2 , were selected to minimize the $L$ 


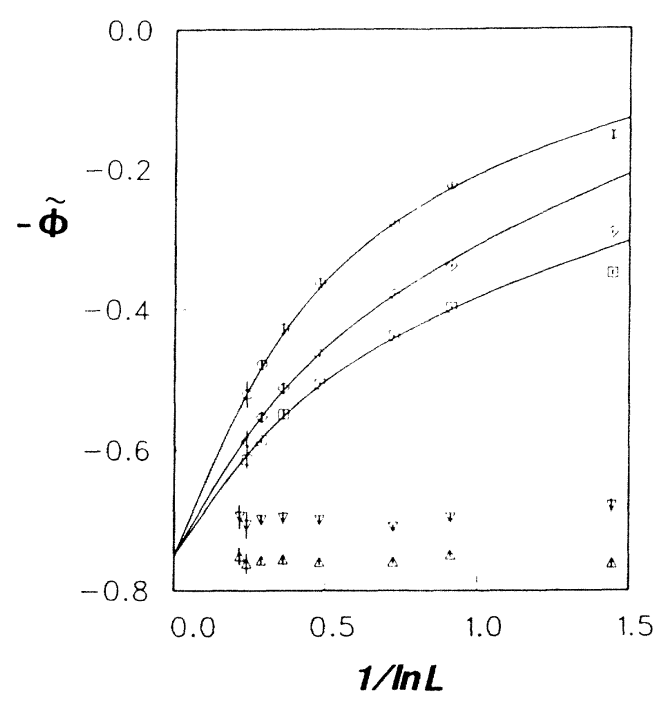

FIG. 3. Estimates of $-\widetilde{\phi}$ versus $1 / \ln L$ for the following slow-bond delay distributions: power law with $k=0.2(\diamond)$, $k=0.5(\square), k=2(O)$, exponential $(\triangle)$, and arctan with $k=2$ $(\nabla)$. Fitted curves are obtained from the correction-to-scaling analysis discussed in the text.

dependence of $\widetilde{\phi}$ to allow reliable extrapolation. Extrapolation gave the estimates $0.76 \pm 0.01$ and $0.70 \pm 0.01$, respectively, for $\widetilde{\phi}$. We interpreted the statistically significant discrepancy between these estimates as a consequence of the fact that for these distributions, $F(x) \sim x^{k}$ is a small- $x$ approximation which is not exact in any neighborhood of $x=0$.

The power-law distribution

$$
F(x)=\min \left\{[k x /(k+1)]^{k}, 1\right\}
$$

is advantageous in this regard, but the computed results exhibit too strong an $L$ dependence to allow reliable extrapolation. Nevertheless, we can use the power-law results to validate Eq. (2.6) indirectly by means of a correctionto-scaling analysis. Namely, we adopt the usual ${ }^{48}$ functional form

$$
\begin{aligned}
v(p) & \sim\left(p_{c}-p\right)^{-\psi}\left[1+\hat{c}_{1}\left(p_{c}-p\right)^{\omega}\right] \\
& \sim \xi^{\tilde{\phi} / k+1}\left(1+c_{1} \xi^{-\omega / v}\right),
\end{aligned}
$$

which defines the correction-to-scaling exponent $\omega .\left(\hat{c}_{1}\right.$ and $c_{1}$ are constants.) Using Eq. (2.2), this form has been expressed in terms of $\xi$. Taking $\xi=L$, we obtain the finite-size scaling formulation

$$
v \sim L^{\tilde{\phi} / k+1}\left(1+c_{1} L^{-\omega / v}\right) .
$$

If we assume that the $L$ dependence of $v$ can be expressed alternatively by means of an $L$-dependent exponent

$$
v \sim\left(p_{c}-p\right)^{-\psi(L)} \sim \xi^{\tilde{\phi}(L) / k+1}=L^{\tilde{\phi}(L) / k+1},
$$

then for large $L$ we obtain

$$
\widetilde{\phi}(L) / k=\widetilde{\phi} / k+\left(c_{2} / \ln L\right)\left[1+\left(c_{1} / c_{2}\right) L^{-\omega / v}\right],
$$

where $c_{2}$ is an additional constant. Here the notation $\widetilde{\phi}(L)$ exhibits the $L$ dependence explicitly in order to dis- tinguish the $L$-dependent exponent defined by Eq. (4.3) for finite $L$ from the value $\widetilde{\phi}$ of the exponent in the limit $L \rightarrow \infty$.

Equation (4.7) closely resembles the finite-size scaling expression for estimation of the correlation-length exponent. ${ }^{46}$ We could use Eq. (4.7) to estimate $\widetilde{\phi}$ for given $F(x)$ by adjusting the parameters $\widetilde{\phi}, \omega_{2} c_{1}$, and $c_{2}$ to fit Eq. (4.7) to the computed results for $\widetilde{\phi}(L)$. Rather than implementing this four-parameter fit to a limited data set, we proceed by fitting Eq. (4.7) to the power-law data of Fig. 3, assuming that Eq. (2.6) is exact, i.e., $\widetilde{\phi}=1 / v\left(=\frac{3}{4}\right.$ for $d=2$ ), and requiring that $\omega$ be universal, i.e., the same for all three $k$ values shown in Fig. 3. The amplitudes $c_{1}$ and $c_{2}$ are taken to be nonuniversal, i.e., they are readjusted for each $k$ value. The curves fitted in this manner are shown in Fig. 3. We obtain $\omega=0.96 \pm 0.01$, where the estimated uncertainty is based on fits in which $\omega$ is allowed to be $k$ dependent.

Despite the number of free parameters in the fit, we regard the excellent agreement which is achieved over a wide range of $L$ values as significant, not only with regard to confirmation of Eq. (2.6), but also with regard to confirmation of the correction-to-scaling analysis. In particular, we have obtained a rather precise estimate of the correction-to-scaling exponent $\omega$, for which no scaling law has yet been proposed. [Note that the finite-size correction-to-scaling analysis based on Eq. (4.5) is distinct from the finite- $(a / b)$ correction to scaling, Eq. (2.15), for which the amplitude as well as the exponent of the correction-to-scaling term were derived in Sec. II B.]

The numerical results cannot exclude the previously proposed scaling law for $\widetilde{\phi}, \mathrm{Eq}$. (2.3), since the difference between the numerical predictions of the two proposals is barely statistically significant. However, we regard Eq. (2.6) as preferable because it has a sounder conceptual basis.

\section{Application to chemical propagation}

The physical interpretation of the convergence condition for the estimate, Eq. (4.2), of the chemicalpropagation exponent is obtained by comparison to the scaling law, Eq. (2.1). We obtain

$$
\phi=\ln \langle\tau \mid \tau=O(b)\rangle / \ln L,
$$

which is consistent with the scaling $\tau_{A B} \sim \xi^{\phi}$, where $A$ and $B$ are now points connected by a path containing only $b$ bonds, so $\tau_{A B}=O(b)$, and where finite-size scaling gives $\xi=L$ at $p=p^{*}$.

Carrying over the reasoning of Sec. IV B, Eq. (4.2) is valid for any distribution of fast-bond time delays, provided that the right-hand side of Eq. (4.8) converges numerically for large $L$. (This might not occur for all distributions.) The chemical-propagation scaling law, Eq. (2.1), may be generalized to such distributions by reinterpreting $\phi$ as a fractal index governing first-passage time rather than chemical distance. As indicated in Sec. II C, no scaling law governing the distribution dependence of $\phi$ has yet been established. In fact, the relationship of $\phi$ to other percolation exponents in the case of identical fast-bond time delays has not yet been established. (Several propo- 
sals which have been offered are discussed in Sec. V C.)

To compute $\phi$, we evaluated the right-hand side of Eq. (4.8) based on Monte Carlo RSRG computations for the chemical-propagation regime, taking $b=1$ and $a=\infty$. The computed results are indicated by squares in Fig. 4 . The number of realizations per data point ranges from 50000 for $L=2$ to 300 for $L=300$. In this instance, the estimates of $\phi$ exhibit strong $L$ dependence. The nonmonotonicity of $\phi(L)$ with respect to $L$ is not unreasonable in view of the finite-size effects discussed earlier, which can introduce spurious couplings as well as omit relevant couplings.

To check the qualitative validity of the results, $\phi(L)$ was recomputed by another method based on an alternative definition of $p^{*}$. So far, we have adopted the usual definition of $p^{*}$ as the fixed point of the ensembleaveraged recursion relation for $p$. Alternatively, we can prepare a realization by starting with an $L \times L$ cell with all time delays equal to $b$, corresponding to $p=1$, and changing randomly chosen bonds to time delay $a$ until a value of $p$ is reached at which the $b$ bonds no longer connect the opposite edges. If we define $p^{*}$ for the realization as the percolation threshold for that realization (based on the connectivity rule stated in Sec. IV A), then value of $\tau$ for the realization is the first-passage time at the smallest value of $p$ for which the $b$ bonds percolate. The computational procedure based on this definition is applicable not only to the square bond lattice but also to lattices for which $p^{*}(L)$ is not known a priori.

This method is computationally costlier than the method used earlier because $b$-bond connectedness must be checked after each bond change, as in the large-cell Monte Carlo RSRG for percolation. ${ }^{41}$ Therefore, fewer realizations were employed than in the earlier computation so the statistical error is greater. Results computed by this method are indicated by circles in Fig. 4. $\phi(L)$ exhibits the same qualitative behavior as before, ${ }^{49}$ and the results obtained by the two methods appear to be converging for large $L$. The strong dependence of $\phi$ on $L$ precludes reliable application of the extrapolation procedure of Sec. IV B.

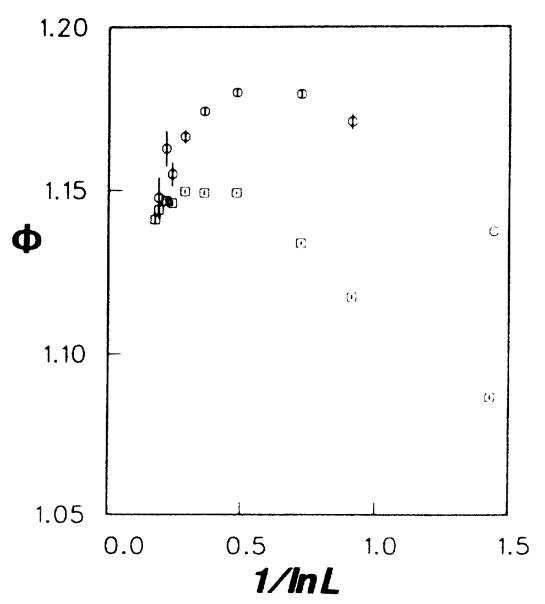

FIG. 4. Estimates of the chemical-distance exponent $\phi$, computed in $L \times L$ cells by two different methods (squares and circles, respectively) defined in the text.

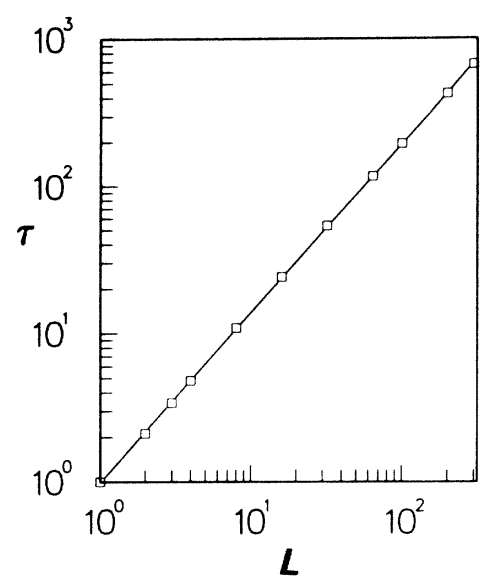

FIG. 5. Mean first-passage time $\tau$ versus cell size $L$ from the computations corresponding to the squares in Fig. 4. The slope of the least-squares fitted line is $\phi=1.148 \pm 0.003$.

In Fig. 5, computed estimates for the expectation value in the numerator of Eq. (4.8) (which were used to obtain the results indicated by squares in Fig. 4) are plotted in the same manner as for previous computations $s^{1,45,50}$ of $\phi$. The slope of a $\log -\log$ plot of this quantity versus $L$ should provide a direct estimate of $\phi$ based on the definition of a fractal index, provided that the range of $L$ values corresponds to the fractal regime. A least-squares fit to the data gives $\phi=1.148 \pm 0.003$, consistent with previous computational results. Although a high degree of linearity on such a plot generally indicates the onset of scaling at relatively low $L$ values, ${ }^{43}$ the conclusion drawn from Fig. 4 is strikingly different. Thus, we regard the results of the large-cell Monte Carlo RSRG computation for $\phi$ as inconclusive. We have therefore obtained ${ }^{14}$ estimates of $\phi$ by an alternate method. The method is outlined in Sec. V A and the estimates of $\phi$ are discussed in Sec. V C.

\section{COMPUTATIONS ON STRIPS}

\section{A. Computational method}

To test the finite- $(a / b)$ scalings predicted in the transition regime, in particular Eq. (2.14) for $v\left(p_{c}\right)$, the computational domain of Sec. IV A could be employed by taking $a$ and $b$ to be finite and estimating $v\left(p_{c}\right)$ by $L /\langle\tau\rangle$, where $\langle\tau\rangle$ is the mean first-passage time between opposite edges of an $L \times L$ box. In this instance, however, the RSRG is not invoked to interpret the results, so there is no inherent advantage in taking the computational domain to be a square box. Instead, we have simulated the propagation process for finite $a / b$ on a long strip of transverse span $L$ (or transverse cross-section $L \times L$, in three-dimensional computations). The computational domain is thus the same as that employed in the transfermatrix ${ }^{13}$ method for conductivity problems. The precision achieved by the transfer-matrix method at relatively low $L$ values indicates that the strip geometry may be advantageous. We have also employed this geometry in computations of propagation exponents for stirredpercolation systems. ${ }^{8,9}$ 
The computations are performed on square and simple-cubic bond lattices. As in the RSRG calculations (Secs. III and IV), each bond on the lattice is randomly assigned time delay $b$ with probability $p_{c}$, otherwise time delay $a>b$, except that here the limit of infinite $a / b$ is not taken. Starting from an edge of $\operatorname{span} L$ for $d=2$ (or a square of area $L^{2}$ for $d=3$ ), the farthest distance $x(t)$ of a site on the propagation front from the starting edge increases monotonically with time. For large $t, x(t) / t$ converges to a constant, the first-passage velocity $v$.

We employ periodic transverse boundary conditions, which avoid cluster-size bias associated with fixed boundary conditions. ${ }^{51,52}$ Although strips of length $3 L$ are used for the computations, periodic longitudinal boundary conditions are applied so that the computational domain is traversed repeatedly, simulating propagation along an infinite strip. New bonds are randomly generated just ahead of the farthest longitudinal advance $x(t)$ of the propagation front whenever $x(t)$ increases. Test cases with longitudinal span larger than $3 L$ confirm that our algorithm is equivalent to propagation on an infinite strip.

\section{B. Verification of scaling laws}

Since we have assumed that all slow bonds have identical time delay $a$, Eq. (2.7) with $k=\infty$ gives $\psi=v$. In conjunction with Eq. (2.1), Eq. (2.14) therefore becomes

$$
v \sim a^{-1}(a / b)^{1 / \phi}
$$

valid for $1 \ll a / b \ll L^{\phi}$, where the upper bound follows from the finite-size scaling arguments of Sec. IIE. For $a / b>L^{\phi}$, Eq. (2.34) gives

$$
v \sim L / a,
$$

where the factor $1 / a$ has been made explicit.

Our computations probe the finite- $(a / b)$ and finite-size scaling regimes by varying the ratio $a / b$. Equations (5.1) and (5.2) are formulated for fixed $a$, with $a / b$ varied by reducing $b$. For simpler processing, we first compute the velocity $v_{b}$ at fixed $b=1$ for various integer values $a>1$ and afterwards rescale according to $v=(a / b) v_{b}$ to obtain the velocity scaled as in Eqs. (5.1) and (5.2).

Each estimate of $v$ is based on five independent repli-

TABLE II. Distance $D_{i}$, in lattice spacings, dedicated to the initial transient, and distance $D$ over which statistics were gathered versus transverse span $L$, for square $(d=2)$ and simplecubic $(d=3)$ lattices.

\begin{tabular}{rcccc}
\hline \hline$L$ & $D_{i}(d=2)$ & $D(d=2)$ & $D_{i}(d=3)$ & $D(d=3)$ \\
\hline 6 & 720 & 7200 & 720 & 6480 \\
8 & 960 & 9600 & 720 & 6480 \\
12 & 1440 & 6480 & 360 & 3600 \\
16 & 960 & 9600 & 192 & 768 \\
24 & 1152 & 10368 & 288 & 1152 \\
32 & 960 & 4800 & 288 & 1056 \\
48 & & & 288 & 864 \\
64 & 1920 & 7680 & & \\
128 & 761 & 3840 & & \\
256 & 2304 & 6912 & & \\
\hline \hline
\end{tabular}

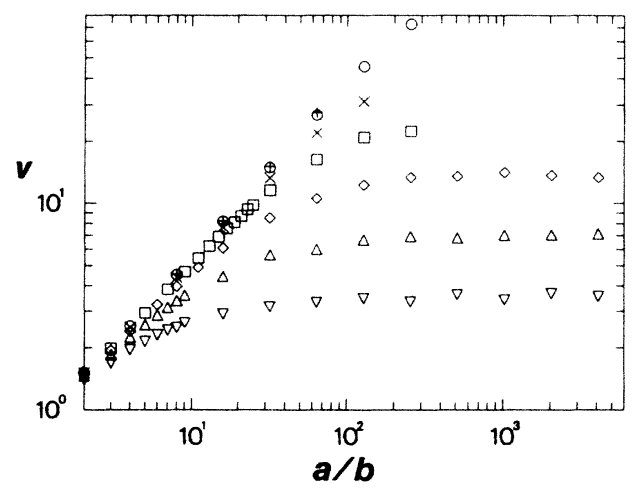

FIG. 6. Estimates of the first-passage velocity $v$ versus the time-delay ratio $a / b$ for a square bond lattice at $p=p_{c}=0.5$ with transverse span $L=2(\nabla), 4(\triangle), 8(\diamond), 16(\square), 32(\times)$, $128(0)$, and $256(+)$.

cate simulations, from which the mean value and the standard deviation of $v$ are extracted. For each replicate, propagation over a longitudinal distance of $D_{i}$ lattice spacings is dedicated to elimination of initial transients, and the first-passage velocity is taken to be the remaining propagation distance, $D=x(t)-D_{i}$, divided by the corresponding passage time. Table II shows values of $D_{i}$ and $D$ for $L$ values used to estimate $\phi$ (Sec. V C).

Figure 6 shows our calculated $v$ as a function of $a / b$ for a square bond lattice at $p_{c}=\frac{1}{2}$ for $L$ values $2(\nabla), 4$ $(\triangle), 8(\diamond), 16(\square), 32(\times), 128(\bigcirc)$, and $256(+)$. For clarity, error bars (which are smaller than the plotting symbols) and data for some additional $L$ values are omitted.

Figure 7 shows similar data emphasizing the finite$(a / b)$ scaling regime for a simple-cubic lattice at $p_{c}=0.2492$ (Ref. 40) for $L$ values $8(\square), 16(\times), 32(O)$, and $48(+)$. The computations clearly confirm the finite- $(a / b)$ and finite-size scalings, Eqs. (5.1) and (5.2), respectively, for $d=2$ and $d=3$.

\section{Estimation of the chemical-distance exponent}

The data in Figs. 6 and 7 provide estimates of the chemical-distance exponent $\phi$. In accordance with the ar-

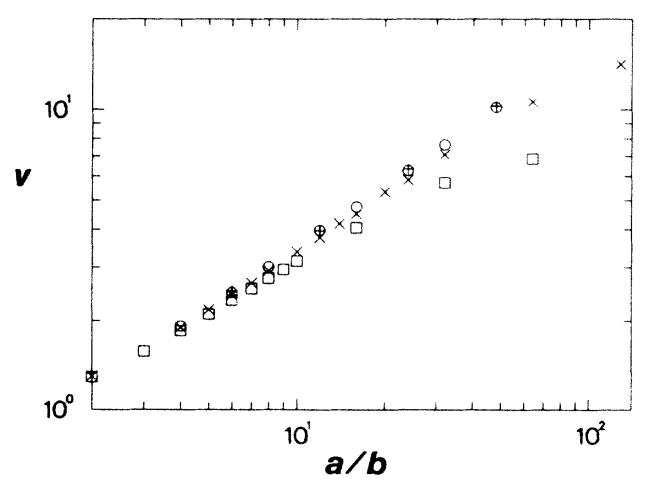

FIG. 7. Estimates of the first-passage velocity $v$ versus the time-delay ratio $a / b$ for a simple-cubic bond lattice at $p=p_{c}=0.2492$ with transverse $\operatorname{span} L=8(\square), 16(\times), 32$ ( $\left.L\right)$, and $48(+)$. 


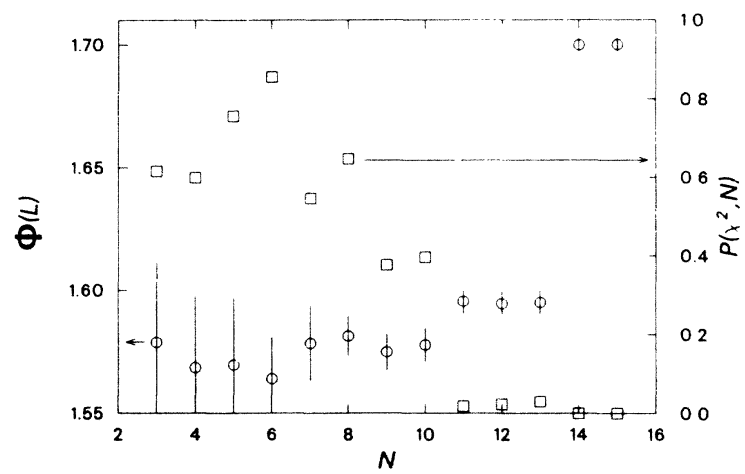

FIG. 8. Values of $P\left(\chi^{2}, N\right)(\square)$, the $\chi^{2}$ distribution evaluated at the weighted sum of squared deviations $\chi^{2}$, and the chemical-distance exponent $\phi(L)(O)$, as a function of the number of fitted points $N$ from Fig. 7 with transverse span $L=16$.

guments in Sec. IV, $\phi$ is the infinite- $L$ extrapolation of a sequence of estimates, $\phi(L)$. By Eq. (5.1), each finite- $L$ estimate, $\phi(L)$, is the reciprocal of the slope in the finite$(a / b)$ regime in Figs. 6 and 7.

Our goodness-of-fit procedure for isolating the finite$(a / b)$ scaling regime exploits the $\chi^{2}$ distribution for $N$ fitted points, $P\left(\chi^{2}, N\right)$, evaluated at the weighted sum of squared deviations for the fit, $\chi^{2}$. As points are removed from either end of a range of points that is initially larger than the scaling regime, $P\left(\chi^{2}, N\right)$ undergoes a transition from values $P\left(\chi^{2}, N\right) \sim 0$ characteristic of poor linear fits to values $P\left(\chi^{2}, N\right) \sim 0.5$ in the finite- $(a / b)$ scaling regime. The inverse slope of a weighted linear least-squares fit through a range of points that is well within the scaling regime gives our estimate of $\phi(L)$.

For example, Fig. 8 shows $P\left(\chi^{2}, N\right)(\square)$ as a function of $N$ for a simple-cubic bond lattice with $L=16$ ( $\times$ in Fig. 7). Evidently, $N \leq 10$ defines the finite- $(a / b)$ scaling regime, as evidenced also by constant values of $\phi(16)(0)$. The value $N=6$, for which $6 \leq a / b \leq 14$, lies well within the finite- $(a / b)$ regime and gives the estimate $\phi(16)=1.56 \pm 0.02$.

Estimates of $\phi(L)$ obtained as above provide the extrapolated estimates ${ }^{14} \phi=1.021 \pm 0.005$ for $d=2$ and $\phi=1.26 \pm 0.06$ for $d=3$, thus raising the possibility that the chemical distance is nonfractal $(\phi \equiv 1)$ for $d=2$. These values favor a scaling law proposed by Roux, ${ }^{53}$ $\phi=(2+d) / 4$, over alternatives proposed by Alexandrowicz, ${ }^{54} \phi=(2+d) /(3+d / 6)$, and by Havlin and Nossal, ${ }^{1} \phi=d-(1+\beta) / v$.

\section{RELATIONSHIP TO STIRRED PERCOLATION}

\section{A. Propagation}

The contact-propagation regime was originally identified in an analysis of stirred-percolation systems. ${ }^{8}$ In the simplest formulation of stirred percolation, the "allowed" region of the lattice at any instant consists of sites occupied by one or more independent random walkers, each of which can move with equal probability to any nearestneighbor site. In a variant of this model, ${ }^{8,55}$ random walkers are mutually avoiding, i.e., a site can be occupied by at most one walker at any instant. In more elaborate models ${ }^{56,57}$ representing the detailed time evolution of physical systems such as microemulsions, the random walkers are partially interpenetrating spheres which are allowed to move in the continuum rather than being confined to a lattice, and spatial correlations are introduced by means of an interaction potential.

The propagation process for stirred percolation is formulated as an irreversible change of state (from "unignited" to "ignited") of the unignited walker(s) on sites adjacent to a site with an ignited walker. This change of state occurs after an "induction time" $b$ during which the unignited and ignited walkers are adjacent. As the terminology suggests, $b$ is analogous to the fast-bond time delay of first-passage percolation. In the limit $b \rightarrow 0$, all walkers within any connected cluster of unignited walkers which comes into contact with an ignited walker are immediately ignited, so the time $\tau$ until the first such contact event becomes rate limiting with respect to propagation of the ignition front (hence the term "contact propagation").

To further develop the correspondence between stirred and first-passage percolation, we consider the distributional properties of the contact time $\tau$ of stirred percolation in the contact-propagation regime $b=0$. If the fraction of occupied sites at any instant is $p \leq p_{c}$, then there are large clusters of ignited (unignited) walkers just behind (ahead of) the ignition front. A pair of adjacent clusters on either side of the front is separated by a layer of $n$ unoccupied sites analogous to the $n$ crossing bonds defined in Sec. II B. Just as the $n$ dependence of the time $\tau_{A B}$ until the first crossing depends on the distributional properties of the slow-bond time delay $a$ (through the parameter $k$ in $\tau_{A B} \sim n^{-1 / k}$ ), the $n$ dependence of the contact time $\tau$ of stirred percolation depends on the temporal random process governing walker motion. For instance, if all walkers move simultaneously at each integer time (a discrete-time process), then for $n \gg 1$, a contact event is almost certain to occur at time $\tau=1$. This is analogous to the firstpassage result $\tau_{A B} \equiv 1$ if all slow-bond time delays are equal to $a=1$. Extending the analogy to a continuoustime process for which the time between successive moves of a given walker is Poisson distributed, the contact time $\tau$ should scale in the same manner as $\tau_{A B}$ for Poissondistributed slow-bond time delays. For the Poisson distribution $F(x)=1-\exp (-\lambda x), \quad k=1$ and therefore $\tau_{A B} \sim 1 / n$. Thus, we obtain $\tau \sim 1 / n$ for the continuoustime stirred-percolation process.

As we noted previously, 8,9 the distinction between discrete-time and continuous-time stirred-percolation processes is crucial because different scalings and therefore different critical exponents are obtained for the two processes, the latter process being more relevant to physical applications. In particular, we evaluate the propagation exponent governing the divergence of $v=\xi / \tau$ as $p \rightarrow p_{c}$, assuming that $n$ scales for stirred percolation in the same manner as for first-passage percolation [Eq. (2.6)]. The results are identical to the corresponding cases of Eq. (2.7), namely, $v \sim\left(p_{c}-p\right)^{-\psi}$, where

$$
\psi=\left\{\begin{array}{l}
v, \text { discrete-time process } \\
v+1, \text { continuous-time process }
\end{array}\right.
$$


For the discrete-time process, this gives $\psi=1.33$ for $d=2$ and $^{39} \psi=0.88$ for $d=3$, compared to the computational results $^{8} 1.48$ for $d=2$ and 0.80 for $d=3$. For the continuous-time process, this gives $\psi=2.33$ for $d=2$, compared to the computational result 2.44 . In view of computational imprecision and possible systematic bias, ${ }^{9}$ we do not regard the differences between the predicted and computed results as significant.

We do not expect that all stirred-percolation processes, including those involving spatial correlations, are in the same universality class. Nevertheless, the success of Eq. (6.1) indicates that the analogy between first-passage percolation and stirred percolation has some quantitative as well as qualitative validity. This suggests that other aspects of the scaling analysis of Sec. II, such as crossover to chemical propagation and finite- $(a / b)$ scaling, may likewise be applicable to stirred percolation. In Sec. VI B, we pursue this analogy further by reconsidering the transport (conductivity) problem ${ }^{8,15,55-58}$ for stirred percolation.

\section{B. Conductivity}

We consider the conductivity of a stirred-percolation system in the limits of infinite charge mobility in the "allowed" region (i.e., sites occupied by one or more random walkers) and zero charge mobility in the "forbidden" region (i.e., unoccupied sites). Thus, the charge density $\rho$ per site immediately equilibrates to a constant value over the cluster whenever two clusters merge to form a single cluster. We assume that the merger of clusters of radius $\xi$ is the dominant charge-transfer mechanism for large $\xi$, and that the frequency of such mergers scales as $1 / \tau$, where $\tau$ is the contact time defined in Sec. VIA. The total charge transfer $Q$ per merger event is proportional to the product of the cluster size $\xi^{d_{f}}$ times the charge-density difference $\Delta \rho$ prior to merging. Assuming that the applied field $E$ is weak, the mean charge-density gradient is constant, so $\Delta \rho \sim \xi E$. Defining the conductivity by means of the relation $\sigma=j / E$, where the current density is $j \sim Q /\left(\xi^{d-1} \tau\right)$, we obtain

$$
\sigma \sim \xi^{d_{f}-d+2} / \tau \sim \xi^{2-\beta / v} / \tau
$$

where we have used the relation $d_{f}=d-\beta / v$ for the fractal dimension of a percolation cluster. ${ }^{32}$

For the discrete-time stirred-percolation process, we showed in Sec. VI A that $\tau=1$, so Eq. (6.2) gives

$$
\sigma \sim\left(p_{c}-p\right)^{\beta-2 v}
$$

identical to the result obtained in previous ${ }^{15,55}$ analyses. For the continuous-time stirred-percolation process, however, we showed that $\tau \sim 1 / n \sim \xi^{-1 / v}$, so that

$$
\sigma \sim\left(p_{c}-p\right)^{\beta-2 v-1}
$$

We expect that the continuous-time result, Eq. (6.4), rather than the discrete-time result, Eq. (6.3), should be applicable to physical processes. Previous analyses ${ }^{15,55}$ omitted this distinction because $\tau$ was taken to be the characteristic time between walker moves, which is of order unity in either case, rather than the time until the first cluster-contact event, which vanishes due to the divergence of the number $n$ of sites at which the event may occur. We therefore regard the reported ${ }^{15,58}$ agreement of Eq. (6.3) with conductivity measurements in microemulsions as unexplained, since we have arrived at Eq. (6.4) using essentially the same reasoning as employed previously, but with a more realistic treatment of the temporal random process governing charge transfer.

One possible explanation is that some of the experimental data used to estimate the conductivity exponent fall within the transition regime associated with the small but finite charge mobility in the "forbidden region," analogous to the transition regime of Sec. II B. Computer simulations ${ }^{57}$ of transport for continuous-time stirred percolation at finite mobility ratios give results in reasonable numerical agreement with the discrete-time scaling, Eq. (6.3). However, analysis of these data based on Eq. (6.4) and considerations analogous to those of Sec. II B indicates that the computed results fall within the transition regime. We therefore anticipate that the apparent agreement with Eq. (6.3) will be supplanted by scaling in accordance with Eq. (6.4) if the computations and the experiments are performed at higher mobility ratios.

A limitation common to the present as well as previous analyses of the conductivity of stirred-percolation systems is the omission of dielectric and capacitive effects which may influence the experimental results. (This omission is implicit in the assumption of uniform charge density throughout a high-mobility cluster.) In view of this and the aforementioned considerations, caution should be exercised in the interpretation of the microemulsion measurements.

\section{SUMMARY}

We have shown that the composition dependence of the first-passage velocity in a disordered medium exhibits features qualitatively similar to those previously identified in studies of transport problems. In a binary medium, the first-passage velocity exhibits a sharp rise near the percolation threshold of the phase in which the intrinsic propagation velocity (the inverse of the individual-bond time delay) is higher. Scaling of the propagation exponents governing the criticality of the first-passage velocity on either side of the percolation threshold has been examined. Corrections to scaling and crossover from the contact-propagation regime, in which the high-velocity phase consists of isolated clusters, to the chemicalpropagation regime, in which the high-velocity phase percolates, have been characterized as a function of the ratio of the intrinsic velocities in the two phases. Computational results obtained by several different methods confirm key scaling predictions.

We have noted that scaling laws for first-passage percolation are applicable, by duality arguments, to two- 
dimensional superfluid flow, and are also applicable to propagation processes in stirred-percolation systems. By analyzing the distinction between discrete-time and continuous-time stirring processes, we have obtained a new scaling law governing transport in stirred-percolation systems.

\section{ACKNOWLEDGMENTS}

The authors would like to thank A. L. R. Bug and Y. Kantor for helpful discussions. This research was supported by the Office of Basic Energy Sciences, U. S. Department of Energy.
1S. Havlin and R. Nossal, J. Phys. A 17, L427 (1984).

${ }^{2}$ A. L. Ritzenberg and R. J. Cohen, Phys. Rev. B 30, 4038 (1984).

${ }^{3}$ S. Havlin, B. Trus, and H. E. Stanley, Phys. Rev. Lett. 53, 1288 (1984).

${ }^{4}$ P. Grassberger, Math. Biosci. 62, 157 (1983).

5J. P. Straley, in Percolation Structures and Processes, Vol. 5 of Annals of the Israel Physical Society, edited by G. Deutscher, R. Zallen, and J. Adler (Hilger, Bristol, 1983), Chap. 15.

${ }^{6}$ A. R. Kerstein, Phys. Rev. B 31, 7472 (1985).

${ }^{7}$ A. Coniglio and H. E. Stanley, Phys. Rev. Lett. 52, 1068 (1984). The "unscreened perimeter" concept introduced in this analysis is discussed in more detail in P. Meakin, H. E. Stanley, A. Coniglio, and P. A. Witten, Phys. Rev. A 32, 2364 (1985).

${ }^{8}$ A. R. Kerstein, Phys. Rev. B 30, 2980 (1984).

${ }^{9}$ A. R. Kerstein, Phys. Rev. B 31, 321 (1985).

10P. M. Kogut and J. P. Straley, J. Phys. C 12, 2151 (1979).

${ }^{11}$ C. Tsallis, A. Coniglio, and G. Schwachheim, Phys. Rev. B 32, 3322 (1985), and references therein.

12T. Keyes, Phys. Rev. A 28, 2584 (1983); T. Ohtsuki and T. Keyes, Phys. Lett. 104A, 77 (1984).

13J. Vannimenus and J. P. Nadal, Phys. Rep. 103, 47 (1984).

${ }^{14}$ B. F. Edwards and A. R. Kerstein, J. Phys. A 18, L1081 (1985).

${ }^{15}$ M. Laguës, J. Phys. (Paris) Lett. 40, L331 (1979).

16J. C. Wierman, Ann. Prob. 10, 509 (1982).

${ }^{17}$ Y. Gefen, A. Aharony, and S. Alexander, Phys. Rev. Lett. 50, 77 (1983).

18P. G. de Gennes, J. Phys. (Paris) Colloq. 41, C3-17 (1980).

${ }^{19}$ R. F. Voss, J. Phys. A 17, L373 (1984), and references therein.

${ }^{20}$ S. S. Wilks, Mathematical Statistics (Wiley, New York, 1962), Chap. 9.

${ }^{21}$ D. C. Wright, D. J. Bergmann, and Y. Kantor, Phys. Rev. B 33, 396 (1986); Y. Kantor (unpublished). Our "crossing bonds" correspond to Kantor's "singly disconnecting bonds."

${ }^{22}$ A. Coniglio, J. Phys. A 15, 3829 (1982).

${ }^{23}$ A. L. Efros and B. I. Shklovskii, Phys. Status Solidi B 76, 475 (1976).

24J. P. Straley, J. Phys. C 9, 783 (1976).

25J. P. Straley, J. Phys. C 13, 819 (1980).

${ }^{26}$ A. Ben-Mizrahi and D. J. Bergman, J. Phys. C 14, 909 (1981).

27J. P. Straley, J. Phys. C 15, 2343 (1982).

28J. P. Straley and S. W. Kenkel, Phys. Rev. B 29, 6299 (1984). Analagous results for deterministic fractal networks of nonlinear resistors are presented in R. Blumenseld and A. Aharony, J. Phys. A 18, L443 (1985).

${ }^{29}$ A. S. Skal and B. I. Shklovskii, Fiz. Tekh. Poluprovodn. 8, 1586 (1974) [Sov. Phys.-Semicond. 8, 1029 (1975)].

30P. G. de Gennes, J. Phys. Lett. (Paris) 37, L1 (1976).

${ }^{31}$ D. A. Huse and R. A. Guyer, Phys. Rev. Lett. 43, 1163 (1979).

32D. Stauffer, Phys. Rep. 54, 1 (1979).
${ }^{33}$ A. Kapitulnik, A. Aharony, G. Deutscher, and D. Stauffer, J. Phys. A 16, L269 (1983).

${ }^{34}$ H. E. Stanley, P. J. Reynolds, S. Redner, and F. Family, in Real Space Renormalization, edited by T. W. Burkhardt and J. M. J. van Leeuwen (Springer, Heidelberg, 1982), Chap. 7.

${ }^{35}$ P. J. Reynolds, W. Klein, and H. E. Stanley, J. Phys. C 10, L167 (1977).

${ }^{36}$ D. C. Hong and H. E. Stanley, J. Phys. A 16, L525 (1983).

37J. P. Straley, J. Phys. C 12, 3711 (1979).

${ }^{38}$ T. Ohtsuki and T. Keyes (unpublished).

${ }^{39}$ D. S. Gaunt and M. F. Sykes, J. Phys. A 16, 783 (1983), and references therein.

${ }^{40}$ S. Wilke, Phys. Lett. 96 A, 344 (1983).

${ }^{41}$ P. J. Reynolds, H. E. Stanley, and W. Klein, Phys. Rev. B 21, 1223 (1980).

${ }^{42}$ P. D. Eschbach, D. Stauffer, and H. J. Herrmann, Phys. Rev. B 23, 422 (1981).

${ }^{43}$ C. J. Lobb and K. R. Karasek, J. Phys. C 13, L245 (1980).

44J. Bernasconi, Phys. Rev. B 18, 2185 (1978).

${ }^{45}$ H. J. Herrmann, D. C. Hong, and H. E. Stanley, J. Phys. A 17, L261 (1984).

${ }^{46} \mathrm{~K}$. Binder and D. Stauffer, in Applications of the Monte Carlo Method in Statistical Physics, edited by K. Binder (Springer, Heidelberg, 1984), Chap. 8.

${ }^{47} \mathrm{G}$. Grimmett and H. Kesten, Z. Wahrsch. Verw. Geb. 66, 335 (1984).

48J. Adler, M. Moshe, and V. Privman, in Percolation Structures and Processes, Vol. 5 of Annals of the Israel Physical Society, edited by G. Deutscher, R. Zallen, and J. Adler (Hilger, Bristol, 1983), Chap. 17. We obtain the $L$ dependence of $v$ from Eq. (4.5) by invoking finite-size scaling. The functional form of the result, Eq. (4.6), is unchanged if we include corrections to finite-size scaling, as in B. Derrida and L. De Seze, J. Phys. (Paris) 43, 475 (1982).

${ }^{49}$ This qualitative behavior was also obtained in unpublished computations by A. L. R. Bug. Similar behavior has been obtained in large-cell Monte Carlo RSRG computations for growth processes, discussed in H. Nakanishi and F. Family, Phys. Rev. A 32, 3606 (1985).

${ }^{50}$ R. Pike and H. E. Stanley, J. Phys. A 14, L169 (1981).

${ }^{51}$ H. J. Herrmann, B. Derrida, and J. Vannimenus, Phys. Rev. B 30, 4080 (1984).

52D. C. Rapaport, J. Phys. A 18, L175 (1985).

53S. Roux, J. Phys. A 18, L395 (1985).

${ }^{54}$ Z. Alexandrowicz, Phys. Lett. 109 A, 169 (1985).

${ }^{55}$ R. Kutner and K. W. Kehr, Philos. Mag. A 48, 199 (1983).

56S. A. Safran, I. Webman, and G. S. Grest, Phys. Rev. A 32, 506 (1985).

${ }^{57}$ G. S. Grest, I. Webman, S. A. Safran, and A. L. R. Bug (unpublished).

${ }^{58}$ S. Bhattacharya, J. P. Stokes, M. W. Kim, and J. S. Huang, Phys. Rev. Lett. 55, 1884 (1985). 